\title{
Effects of Plant Growth Promoting Rhizobacteria Microbial Inoculants on the Growth, Rhizosphere Soil Properties, and Bacterial Community of Pinus sylvestris var. mongolica Annual Seedlings
}

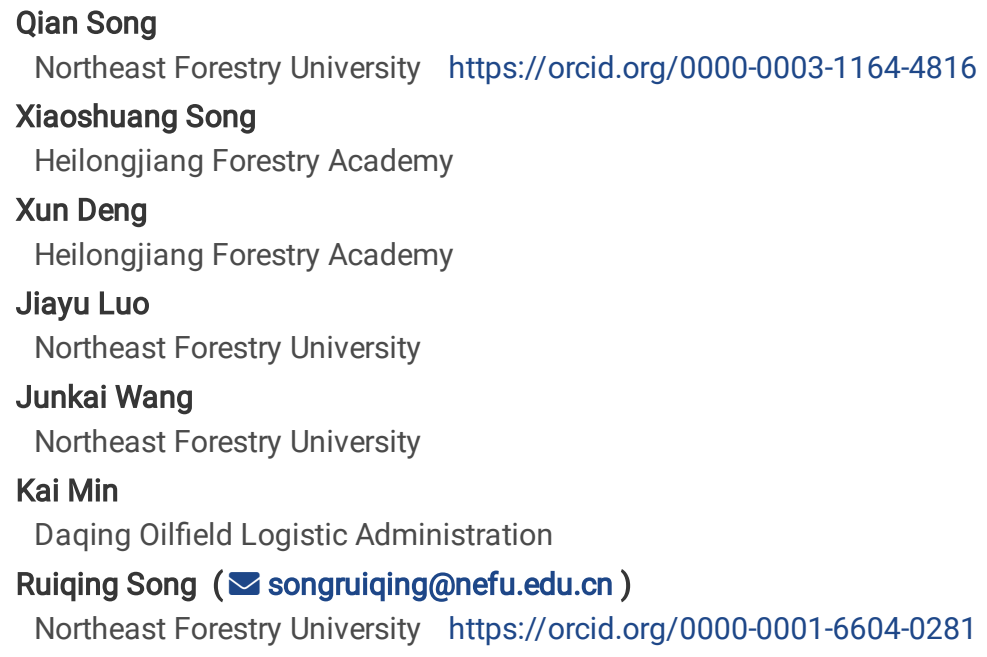

Original article

Keywords: Rhizosphere, Pinus sylvestris var. Mongolica, 16S rRNA, Microbial diversity, Community structure

Posted Date: September 30th, 2020

DOI: https://doi.org/10.21203/rs.3.rs-80682/v1

License: (1) This work is licensed under a Creative Commons Attribution 4.0 International License. Read Full License 


\section{Abstract}

[Objective] Determine the ability of three plant growth promoting rhizobacteria (PGPR) strains (Pseudomonas Mandelli A36, Serratia plymuthica A13 and Pseudomonas koreensis A20) to promote plant growth, evaluate the effect of inoculation with PGPR strains on seedling biomass, root structure, nutrient index, and enzyme activity, and assess the effect of PGPR inoculation on soil nutrient index, enzyme activity, and the soil microecological environment.

[Method] The ability of the three PGPR strains to secrete indole-3-acetic acid (IAA), dissolve inorganic phosphorus, and produce siderophore and hydrolase was determined by the medium color change method, pot experiment to determine the effects of three PGPR strains on plant biomass, physicochemical properties, soil physicochemical properties and microbial diversity.

[Result] The three PGPR strains had the ability to secrete IAA, solubilize inorganic phosphorus, and produce siderophore, the results of the pot experiment showed that inoculation with PGPR strain had a significant effect on plant biomass, root index, nutrient index and enzyme activity, as well as soil nutrient index, enzyme activity and bacterial diversity.

[Conclusion] This study provides basic data references for PGPR strains to improve the soil microecological environment and promote the growth and development of Pinus sylvestris var. Mongolica seedlings.

\section{Introduction}

Soil microorganisms promote material cycling and energy flow in the ecosystem. They play important roles as both producers and decomposers in the ecosystem (Wu et al. 2013; Zhang and Yu, 1990). In addition, soil microorganisms perform processes such as oxidation, nitrogen fixation, nitrification, and ammoniation in the soil to promote the decomposition of soil organic matter and nutrient conversion. Soil microorganisms are widely distributed in the plant rhizosphere and are most speciose in a dynamically changing environment. In 1904, the German scientist Lorenz Hiltner first proposed the concept of the "rhizosphere" which refers to the soil around the root system. In the rhizosphere, plant root activity alters the physical and chemical properties of the soil, providing a special ecological environment for interaction between plants and soil microorganisms (Compant et al. 2010; Kloepper et al. 1980; Liu 2005). Since the concept of the "rhizosphere" was proposed, there have been increasing numbers of studies on the plant rhizosphere, mainly involving the physiological structure of the root system, rhizosphere soil nutrients, rhizosphere soil enzyme activities, and rhizosphere soil microorganisms, as well as the connection between them (Li 2002). There are a large number of active microorganisms, such as fungi, bacteria, and actinomycetes, in the rhizosphere soil, approximately 28-48 times higher than in non-rhizosphere soil. There are about 104-106 fungi genera in every $1 \mathrm{~g}$ of soil, and the main groups include filamentous fungi such as Penicillium, Fusarium, Aspergillus, and Trichoderma (Barreto et al. 2008; I. G et al. 2002; Sreevidya et al. 2016). There are about 104-108 actinomycetes genera in every $1 \mathrm{~g}$ of soil, primarily composed of Streptomyces, Micromonospora, and Nocardia (Cocking 2003). Bacteria are the most abundant rhizosphere soil microorganisms, with each $1 \mathrm{~g}$ of soil containing approximately 106-1010 bacteria genera. The main bacterial groups include Bacillus, Pseudomonas, Flavobacterium, Serratia, Rhizobium, and Azotobacter. Rhizosphere bacteria can promote plant growth, increase plant biomass, promote the absorption and utilization of soil nutrients by plants, improve the microecological environment of the rhizosphere soil, and inhibit or antagonize pathogenic bacteria (Vessey 2003). In addition, PGPR can degrade pollutants in the soil, improve soil fertility, control pests and diseases, and reduce the environmental pollution and soil compaction caused by the use of pesticides and fertilizers (Zhang et al. 2013). One study found that Brevibacillus brevis DZQ3 could significantly promote the growth of tobacco (Zhu et al. 2012), while another discovered that Azospirillum could promote the growth of corn (Zhu et al. 2012). A further study found that Pseudomonas CBT1, CBT6, and Cbt7 has a certain control effect on cucumber fusarium wilt (Yue and Zhang, 2009). Although PGPR has a significant impact on plant growth, the influence of PGPR on perennial tree species and their associated rhizosphere communities are still poorly investigated, and the number of related PGPR reported is also very limited. At present, Bacillus and Pseudomonas are the two most studied and most important plant growth-promoting bacteria (Myresiotis et al. 2012). In addition to these two genera, Serratia has also been reported on, though not extensively so. Serratia sp. sy 5 was found to increase the biomass of corn (Koo and Cho 2009). Serratia sp. CDP-13 could enhance the induced resistance and salt tolerance of wheat (Singh and Jha 2016), while Serratia Sp. A21-4 could promote the growth and development of capsicum and also demonstrated strong rhizosphere colonization ability (Yayou et al. 2016).

Pinus sy/vestris var. mongolica is a geographical variety of Pinus sy/vestris. It has a developed root system and can fully absorb and utilize water. Due to its fast growth, cold resistance, and drought resistance, as well as its aesthetic qualities, it has been widely introduced into the three northern areas of China where it is the main tree species used in shelter and sand control engineering (Zhang and Li, 2003). However, due to the large-scale use of pesticides and chemical fertilizers in recent years, $P$. sy/vestris var. mongolica populations have declined in many areas, causing great economic loss. The introduction of rhizosphere microorganisms to replace pesticides and chemical fertilizers could address this decline as well as promote the growth and development of $P$. sy/vestris var. mongolica, improve its root structure, and enhance its resistance to stress. The application of PGPR is pollution-free, residue-free, and more conducive to human and animal safety (Fiorentino et al. 2018; Zhang et al. 2018). After the introduction of rhizosphere microorganisms, the population and distribution of soil microorganisms will change, the basic physical and chemical properties of the soil will be altered, and the growth and development of plants will also be affected. As different

Page 2/21 
rhizosphere microorganisms have different effects on the soil, it is essential that the impacts of rhizosphere microorganisms on the community structure, species composition, spatial distribution, and diversity of the soil microecological environment are explored.

Therefore, the objectives of this study were to

1. Determine the ability of three PGPR strains (P. mandelii A36, P. koreensis A20, and S. plymuthica A13) to promote plant growth;

2. Evaluate the effect of inoculation with PGPR strains on seedling biomass, root structure, nutrient index, and enzyme activity; and

3. Assess the effect of PGPR inoculation on soil nutrient index, enzyme activity, and the soil micro-ecological environment.

Our overall aim was to explore the use of rhizobacteria in changing the community structure of soil microorganisms, promoting the growth and development of $P$. sy/vestris var. mongolica, and improving the stress resistance of $P$. sy/vestris var. mongolica.

\section{Materials And Methods}

\subsection{Identification of PGPR strains}

The three PGPR strains A13, A36, and A20 were isolated from the rhizosphere soil collected from a P. sylvestris var. mongolica forest at the Zhanggutai Experimental Forest Farm in Liaoning Province $\left(42^{\circ} 43^{\prime}-42^{\circ} 51^{\prime} \mathrm{N}, 121^{\circ} 53^{\prime}-122^{\circ} 22^{\prime} \mathrm{E}\right)$, China. These three strains are highly efficient strains with multiple growth-promoting characteristics. Analysis of $16 \mathrm{~S}$ rRNA sequences was used to identify the three strains. The three strains were inoculated in nutrient broth (NB) (peptone 1\%; beef extract; $1 \%$; sodium chloride $0.5 \%$, Haibo Biotechnology, China) liquid medium and incubated at $37^{\circ} \mathrm{C}$ with shaking (180 rpm) for $24 \mathrm{~h}$, following which the genomic DNA was extracted from each strain using the bacterial genomic DNA isolation Kit DP302 (Beijing Tiangen Biochemical Technology Co., Ltd., China). The 16S rRNA gene primers 27F (5'agagttgatcctggctcag-3') and 1541R (5'-aaggaggtgatcccacgcca-3') were used for amplification (Galkiewicz and Kellogg, 2008). The polymerase chain reaction (PCR) was performed in 25- $\mu \mathrm{L}$ reactions containing $12.5 \mu \mathrm{L} 2 \times$ Taq PCR mix solution, $1 \mu \mathrm{L}$ forward primer, $1 \mu \mathrm{L}$ reverse primer, 1 $\mu \mathrm{L}$ template DNA, and $9.5 \mu \mathrm{L}$ double-distilled $\mathrm{H}_{2} \mathrm{O}$. The reaction conditions were: $94^{\circ} \mathrm{C}$ for $1 \mathrm{~min}, 30$ cycles of $94^{\circ} \mathrm{C}$ for $20 \mathrm{~s}, 56{ }^{\circ} \mathrm{C}$ for $20 \mathrm{~s}$, and $72{ }^{\circ} \mathrm{C}$ for $2 \mathrm{~min}$, followed by a final extension at $72{ }^{\circ} \mathrm{C}$ for $5 \mathrm{~min}$. The reaction products were qualified by $0.8 \%$ agarose gel electrophoresis and then sent to Ruibo Biotech Co., Ltd (Harbin, China) for sequencing. The NCBI Blast server (http://www.ncbi.nlm.nih.gov) was used to compare the sequences with the registered sequences in the GenBank database. A phylogenetic tree was constructed using the maximum likelihood method in the software package MEGA (version 6.0), and the topology of the phylogenetic tree was evaluated using 1,000 bootstrap replicates.

\subsection{Determination of the plant growth promotion characteristics of the PGPR strains}

The molybdenum blue colorimetric method was used to determine the ability of the strains to dissolve inorganic phosphorus (Shekhar 1999). Specifically, the strain suspension was spot-inoculated on sterile National Botanical Research Institute phosphate (NBRIP) solid medium and cultured at $28^{\circ} \mathrm{C}$ for $3 \mathrm{~d}$, during which the formation of a transparent ring was observed. The strains producing transparent circles were inoculated in sterile NBRIP liquid medium and cultured at $28^{\circ} \mathrm{C}$ with shaking $(180 \mathrm{rpm})$ for $7 \mathrm{~d}$. A non-inoculation control was set, and then the soluble phosphorus content of the strain culture solution was measured.

The ability of the strains to produce indole-3-acetic acid (IAA) was tested using the methods of Bric (1991) and (Bric et al. 1991). The strain was inoculated in $\mathrm{R}_{2} \mathrm{~A}$ liquid medium ( $0.5 \mathrm{~g}$ yeast, $0.5 \mathrm{~g}$ peptone, $0.5 \mathrm{~g}$ casein, $0.5 \mathrm{~g}$ glucose, $0.5 \mathrm{~g}$ soluble starch, $0.3 \mathrm{~g} \mathrm{~K}_{2} \mathrm{HPO}_{3}, 0.05 \mathrm{~g} \mathrm{MgSO}_{4}, 0.3 \mathrm{~g}$ sodium pyruvate, and $1000 \mathrm{~mL} \mathrm{H}$ O) supplemented with $200 \mathrm{mg} / \mathrm{L}$ L-tryptophan and incubated at $28^{\circ} \mathrm{C}$ with shaking $(180 \mathrm{rpm})$ for $4 \mathrm{~d}$, following which the strain culture was centrifuged at $12,850 \times g$ for $10 \mathrm{~min}$. One milliliter of the culture solution was used to determine the $\mathrm{OD}_{600}$ value. The same volume of Salkawaski's reagent ( $50 \mathrm{~mL} \mathrm{35 \%} \mathrm{HClO}_{4}+1 \mathrm{~mL} 0.5 \mathrm{M} \mathrm{FeCl}_{3}$ ) was added, and the solution was placed in the dark for $30 \mathrm{~min}$. A color change to red indicated that the strain had produced IAA. The IAA content of the sample was measured at $\mathrm{Od}_{530}$, and the yield was calculated as $\left(\mathrm{mg} \cdot \mathrm{L}^{-1} \cdot \mathrm{OD}_{600}{ }^{-1}\right)=\mathrm{OD}_{600} / \mathrm{OD}_{530}$. All tests were conducted in triplicate.

The ability of the strain to act as an iron-producing carrier was assessed using the improved Chrome Azurol S (CAS) test plate (Shin et al. 2001). First, Mannitol Salt Agar (MSA) medium $\left(0.5 \mathrm{~g} \mathrm{KCl}, 0.5 \mathrm{gMgSO}_{4}, 4 \mathrm{~g}\right.$ glucose, $5 \mathrm{~g}$ casein peptone, $15 \mathrm{~g}$ agar, and $1 \mathrm{~L}$ distilled water), chromeazurol $\mathrm{S}$ dye solution ( $1 \mathrm{mM} \mathrm{CAS}, 0.1 \mathrm{mM} \mathrm{FeCl}_{3}$, and $4 \mathrm{mM}$ cetyltrimethylammonium bromide), and phosphate solution ( $0.5905 \mathrm{~g}$ $\mathrm{NaH}_{2} \mathrm{PO}_{4} \cdot 2 \mathrm{H}_{2} \mathrm{O}, 2.427 \mathrm{~g} \mathrm{Na}_{2} \mathrm{HPO}_{4} \cdot 12 \mathrm{H}_{2} \mathrm{O}, 0.25 \mathrm{~g} \mathrm{NH}_{4} \mathrm{Cl}, 0.075 \mathrm{~g} \mathrm{KH}_{2} \mathrm{PO}_{4}, 0.125 \mathrm{~g} \mathrm{NaCl}$, and $100 \mathrm{~mL}$ distilled water) were prepared, and then the three solutions were sterilized at $115^{\circ} \mathrm{C}$ for $25 \mathrm{~min}$. Before pouring the medium, $50 \mathrm{~mL}$ of CAS dye solution and $50 \mathrm{~mL}$ phosphate solution were added into $1000 \mathrm{~mL}$ MSA medium and mixed well to produce the MSA-CAS detection medium (Chen 2006; Pérez-Miranda et al. 2007; Xiang 2006). The strain suspension was inoculated on the MSA-CAS detection plate and cultured at $28^{\circ} \mathrm{C}$ for $7 \mathrm{~d}$, and a non-inoculation control was set. If an orange-yellow circle formed on the blue CAS plate, it indicated that the strain had produced siderophore. 
The ability of the strain to produce HCN was measured using the improved method of Kloepper et al. (Kloepper et al. 1991). The strain was inoculated on King's B medium supplemented with $4.4 \mathrm{~g} / \mathrm{L}$ glycine $\left(20 \mathrm{~g}\right.$ peptone, $1.5 \mathrm{~g} \mathrm{~K}_{2} \mathrm{HPO}_{4}, 1.5 \mathrm{~g} \mathrm{MgSO} \cdot 7 \mathrm{H}_{2} \mathrm{O}, 10 \mathrm{~g} \mathrm{glycerol}, 15 \mathrm{~g}$ agar, and $1000 \mathrm{~mL}$ distilled water). A filter paper strip soaked in picric acid solution ( $2.5 \mathrm{~g}$ picric acid, $12.5 \mathrm{~g} \mathrm{Na}_{2} \mathrm{CO}_{3}$, and $1000 \mathrm{~mL}$ distilled water) was placed on top of the plate cover and cultured at $28^{\circ} \mathrm{C}$ for $3 \mathrm{~d}$. The change in the color of the filter paper strip from yellow to brown to red indicated the generation of HCN. The intensity of the color was visually recorded.

The ability of the strain to produce hydrolase was measured using the method of Cappuccino and Sherman (Cappuccino 2010). The strain suspension was inoculated on skim milk agar medium (100 g skim milk, $5 \mathrm{~g}$ peptone, $15 \mathrm{~g}$ agar, and $1000 \mathrm{~mL}$ distilled water) and starch agar medium (10 g soluble starch, $5 \mathrm{~g}$ peptone, $3 \mathrm{~g}$ beef extract, $15 \mathrm{~g}$ agar, and $1000 \mathrm{~mL}$ distilled water) and cultured at $28{ }^{\circ} \mathrm{C}$ for $3 \mathrm{~d}$ to observe any transparent areas around the spots. The medium was prepared by adding $1 \%$ cellulose, $0.5 \%$ chitin, and $1 \%$ pectin to the basic medium ( $1 \mathrm{~g}$ glucose, $0.5 \mathrm{~g}$ yeast extract, $0.5 \mathrm{~g} \mathrm{MgSO}_{4}, 1 \mathrm{~g} \mathrm{KCl}, 1 \mathrm{~g} \mathrm{NaNO}_{3}, 1 \mathrm{~g} \mathrm{~K}_{2} \mathrm{HPO}_{4}, 15 \mathrm{~g}$ agar, and $1000 \mathrm{~mL}$ distilled water), and the strain suspension was inoculated into cellulose medium, chitin medium, and pectin medium at $28{ }^{\circ} \mathrm{C}$ for $5 \mathrm{~d}$. The cellulose medium was soaked with $0.01 \%$ Congo red solution for $15 \mathrm{~min}$, following which the solution was poured out and decolorized with $1 \% \mathrm{NaCl}$ solution for 5 min to observe whether any transparent areas appeared on the red background. Gram iodine liquid was then poured into the chitin medium and pectin medium, and the appearance of clear areas on the dark blue background was observed.

\subsection{Pot experiment}

The seeds of $P$. sylvestris var. mongolica (purchased from the Zhanggutai Experimental Forest Farm in Zhangwu County, Liaoning Province, China) were surface-sterilized with potassium permanganate $(0.5 \%, \mathrm{v} / \mathrm{v})$ for $30 \mathrm{~min}$ and then washed five times with sterile distilled water. They were then germinated on sterile moistened gauze at $25^{\circ} \mathrm{C}$ for $5 \mathrm{~d}$. After germination, the seedlings were transferred to plastic pots $(15 \times 15 \mathrm{~cm}$, 20 seeds per pot) filled with a sterile culture substrate of peat soil/vermiculite/sand $(2: 1: 1, \mathrm{v} / \mathrm{v} / \mathrm{v})$. The pots were kept under greenhouse conditions (day/night thermal regime of $22 / 30 \pm 3{ }^{\circ} \mathrm{C}$, and $14 \mathrm{~h}$ light/10 h dark photoperiod) and watered every $2 \mathrm{~d}$ for $14 \mathrm{~d}$, after which the seedlings were inoculated with the bacterial inoculum (Qi et al. 2019).

Before plant inoculation, the three strains were separately inoculated into $250 \mathrm{~mL}$ Erlenmeyer flasks containing $100 \mathrm{~mL}$ of $\mathrm{NB}$ liquid medium and maintained in $28^{\circ} \mathrm{C}$ with shaking $(180 \mathrm{rpm})$ for $48 \mathrm{~h}$. The bacterial cultures were centrifuged at $8000 \mathrm{~g}$ for $10 \mathrm{~min}$ at $4{ }^{\circ} \mathrm{C}$, and the collected bacteria were repeatedly centrifuged, washed with sterilized water three times, and then diluted with sterilized water to achieve an $\mathrm{OD}_{600}$ of 0.6 , which was the final volume ratio used as the inoculum.

For all treatments, including the control, 10 pots (15 seedlings per pot) were prepared, giving a total of 150 seedlings per treatment. There were four treatments: (1) inoculation with sterile water (CK); (2) inoculation with A13; (3) inoculation with A20; and (4) inoculation with A36. The inoculations were performed by transferring $100 \mathrm{~mL}$ of the bacterial inoculum into the planting hole (Yang et al. 2019), where it was introduced at the root system level. The control plants were inoculated with $100 \mathrm{~mL}$ of sterile water. All treatments were arranged at random under the greenhouse conditions given above.

\subsection{Sampling and biomass analysis of the seedlings}

Samples were taken at three months after seedling inoculation. Fifty seedlings from each treatment group were randomly selected, of which 30 were used to measure biomass index, including seedling height (SH), ground diameter (GD), fresh weight of seeding (SFW), dry weight of seeding (SDW), fresh weight of root (RFW), and dry weight of root (RDW). During sampling, damage to the root system of the seedlings was minimized. The root system was washed to remove the soil, following which 10 randomly selected seedlings were used for scanning and grading the root system using an Epson v 700 root scanner. The indexes of root length, surface area, average diameter, number of root tips, bifurcation number, and root volume were analyzed.

\subsection{Nutrient and physiological parameter analysis of the seedlings}

Ten seedlings of $P$. sylvestris var. mongolica were randomly selected for drying. After drying, the roots, stems, and leaves were ground separately and stored in test tubes at room temperature for future use. Total nitrogen (TN) was determined using the Kjeldahl method, and available nitrogen (AN) was determined using the alkaline hydrolysis diffusion method (LY/T 1228-2015). Total phosphorus (TP) was determined by Mo-sb anti-colorimetry, and available phosphorus (AP) was determined using the sodium bicarbonate extraction method (LY/T 1232-2015). Total potassium (TK) was determined by flame photometry, and available potassium (AK) was determined using the NH4OAc leaching flame photometer method (LY/T 1234-2015). Organic matter (OM) was determined using the potassium dichromate oxidationexternal heating method (LY/T 1237-1999). Superoxide dismutase (SOD), catalase (CAT), peroxidase (POD), malondialdehyde (MDA), plantsoluble sugar (PSS) and proline (PRO) were determined using a kit from the Nanjing Jiancheng Bioengineering Company (Patterson et al. 1984).

\subsection{Soil enzyme activities and physiochemical properties analysis}

Page $4 / 21$ 
Upon collection of the plants, the topsoil was removed, and the rhizosphere soil was collected and sieved with a 20-mesh screen. Three replicates were tested in each treatment group. Ten grams of rhizosphere soil was obtained from each treatment group and stored at $4{ }^{\circ} \mathrm{C}$ for the determination of rhizosphere soil enzyme activity. Fifteen grams of soil from each treatment group was divided into three test tubes on average and stored at $-80^{\circ} \mathrm{C}$ for measuring soil microbial diversity. The rest of the rhizosphere soil was air-dried and stored at room temperature for measuring rhizosphere soil nutrients. The determination methods of soil TN, AN, TP, AP, TK, AK, and OM were the same as those described in 2.5. The soil acid phosphatase (APA), catalase (CA), sucrase (SA), and urease (UA) activities were measured using the Nanjing Jiancheng Biological Engineering Company kit.

\subsection{Bacterial diversity analysis}

Three replicate rhizosphere soil samples of the $P$. sylvestris var. mongolica seedlings from each treatment group were sieved with a 10 -mesh screen and stored in a refrigerator at $-80^{\circ} \mathrm{C}$ for high-throughput sequencing. High-throughput sequencing was performed by Majorbio Bio-Pharm Technology Co., Ltd (Shanghai, China) on the Illumina Miseq Sequencer platform. FLASH (version 1.2.11) software was used to splice the paired-end sequences (https://ccb.jhu.edu/software /FLASH/index. shtml) (Magoc and Salzberg, 2011). Usearch (version 7.0) was used to count operational taxonomic unit (OTU) (http://www. drive5.com /usearch/). At the same time, Qiime (version 9.1) was used to duplicate the filters and classify the sequences. The $\beta$ diversity distance was also calculated (http://qiime.org/install/index.html) (Caporaso et al. 2010). Uparse (version 7.0.1090) was used to cluster the sequences with a threshold of 97\% similarity (http://www.drive5.com/uparse/) (Edgar 2013). RDP Classifier (version 2.2) was used to annotate the sequences using the default confidence threshold of 0.7 (http://sourceforge.net/projects/rdp- classifier/).

\subsection{Data processing and analysis}

Data were processed using Excel 2013 software, one way analysis of variance (ANOVA) of IBM spss19.0 software was used to test the significant differences of plant biomass, root structure, nutrient index, enzyme activity, soil nutrient index, enzyme activity, and relative abundance of different bacterial taxonomic levels. The Duncan test separates the mean when $p<0.05$, expressed as the mean \pm standard deviation. At the same time, Pearson correlation analysis of spss19.0 software was used to test the relationship between environmental factors, a diversity index and dominant bacteria. Mothur (version 1.30.2) software was used to analyze the a diversity index (https://www.mothur.org/wiki/ Download_mothur) (Uroz et al. 2016), and the R 'language' software package was used to produce the Venn diagram, bar diagram, Heatmap diagram and PCoA diagram. Circos-0.67-7 software was used to analyze the proportion of dominant species in each sample. The Vegan package in the R statistical program was used to for the redundancy analysis (RDA).

\subsection{Accession number(s)}

The 16S rRNA gene sequences of the three strains were submitted and stored in the GenBank database with the accession numbers MT280201 (Pseudomonas koreensis A20), MT280202 (Serratia plymuthica A13), and MT280203 (Pseudomonas mandelii A36).

\section{Results}

\subsection{Identification of PGPR strains and plant growth-promoting characteristics}

The 16S rRNA gene sequences of the three strains were analyzed, and phylogenetic trees were constructed (Fig. 1). The similarity between strain A13 and Serratia plymuthica DSM 4540 (AJ233433) was 99.00\%, and thus the strain was preliminarily identified as Serratia plymuthica. The similarity between strain A36 and Pseudomonas mandelii (AF058286) was 99.72\%, and thus the strain was preliminarily identified as Pseudomonas mandelii. The similarity between strain A20 and Pseudomonas koreensis Ps 9-14 (AF468452) was 98.80\%, and thus the strain was preliminarily identified as Pseudomonas koreensis.

The three strains had various plant growth-promoting properties (Table 1). Strains A36, A13, and A20 were able to dissolve inorganic phosphorus, and the solubility of the inorganic phosphorus was $189.50 \mu \mathrm{g} / \mathrm{mL}, 211.00 \mu \mathrm{g} / \mathrm{mL}$, and $429.50 \mu \mathrm{g} / \mathrm{mL}$, respectively, and thus strain A13 exhibited the highest inorganic phosphorus solubility of $429.50 \mu \mathrm{g} / \mathrm{mL}$. Strains A36, A13, and A20 all had the ability to secrete IAA. The IAA content of the three strains was $18.88 \mu \mathrm{g} / \mathrm{mL}, 4.59 \mu \mathrm{g} / \mathrm{mL}$, and $22.66 \mu \mathrm{g} / \mathrm{mL}$, respectively. All three strains could produce siderophore. Strains A36 and A20 produced light yellow circles on the CAS detection plate, while strain A13 produced orange-yellow circles. The filter paper strips of strains A13 and A20 changed from yellow to brown after $24 \mathrm{~h}$ of inoculation, and from brown to red after $60 \mathrm{~h}$ of inoculation. The filter paper strip of strain A36 remained unchanged, indicating that strains A13 and A20 had the ability to produce HCN, while A36 did not.

As indicated in Table 1, the three strains produced transparent areas on skim milk agar medium and starch agar medium, indicating that all three strains could produce protease and amylase. Strains A36 and A20 produced transparent areas on the cellulose medium, indicating that these two strains had the ability to produce cellulose. With the exception of strain A36, which was negative on the chitin medium, the remaining strains were positive and could produce chitinases. All three strains produced clear areas against the dark blue background of the pectin medium, indicating that all three strains had the ability to produce pectinase.

Page 5/21 
Table 1

Plant growth-promoting characteristics of the three strains

\begin{tabular}{|c|c|c|c|c|c|c|c|c|c|}
\hline \multirow[t]{2}{*}{ Strains } & \multicolumn{4}{|c|}{ Hydrolytic enzyme production } & \multicolumn{4}{|c|}{ Growth promoting traits } & \multirow{2}{*}{$\begin{array}{l}\mathrm{HCN} \\
\text { production }\end{array}$} \\
\hline & Protease & $\begin{array}{l}\text { a- } \\
\text { amylase }\end{array}$ & Cellulase & Chitinase & Pectinase & $\begin{array}{l}\text { IAA } \\
\text { Production }\end{array}$ & $\begin{array}{l}\text { P } \\
\text { solubilized }\end{array}$ & $\begin{array}{l}\text { Siderophores } \\
\text { production }\end{array}$ & \\
\hline A36 & + & + & + & - & + & + & + & + & - \\
\hline A13 & + & + & - & + & + & + & + & + & + \\
\hline A20 & + & + & + & + & + & + & + & + & + \\
\hline
\end{tabular}

\subsection{Effects of PGPR on seedlings biomass and root structure}

Inoculation with PGPR microbial inoculum influenced the growth characteristics of the P. sy/vestris var. mongolica seedlings (Fig. 2a).

Compared with CK, after inoculation with A13, SH, SFW, RFW and SDW increased the most, increasing by $27.60 \%, 76.19 \%, 46.55 \%$ and $22.86 \%$ respectively $(p<0.05)$. After inoculation with A20, GD increased by up to $22.99 \%(p<0.05)$, after inoculation with A36, RDW increased by up to $30.23 \%(p<0.05)$.

Root length (RL), root volume (RV) and surface area (SA) are important parameters for plant root distribution. The average diameter (AD), the number of root tips (TIPS) and the number of branches (BN) are important parameters for measuring root absorption efficiency. Inoculation with PGPR had a certain effect on the root structure of $P$. sylvestris var. mongolica (Fig. $2 \mathrm{~b}, \mathrm{c}$ ). Inoculation with $\mathrm{A} 20$ had the greatest effect on the root system, with the greatest increase in $\mathrm{RL}, \mathrm{SA}, \mathrm{AD}$, and $\mathrm{BN}$, increasing by $47.88 \%, 63.21 \%, 10.81 \%$ and $57.20 \%$ respectively $(p<0.05)$, inoculation of $A 13$ increased $B N$ by up to $30.61 \%$, and inoculation of $A 36$ increased RV by up to $75.00 \%$.

\subsection{Effects of PGPR inoculation on seedlings and soil nutrients.}

$\mathrm{OM}$ and $\mathrm{N}, \mathrm{P}$, and $\mathrm{K}$ are essential nutrients for plant growth. Inoculation with PGPR had a certain effect on the nutrient content of roots, stems and leaves of $P$. sy/vestris var. mongolica seedlings (Fig. 3a). The content of OM and TN in the roots of inoculation with A36 increased the most, $52.79 \%$ and $21.36 \%$, respectively $(p<0.05)$, the TP content of roots inoculation with A13 increased the most by $28.94 \%(p<0.05)$; The content of $\mathrm{OM}$ and TN in the stems of inoculation with A36 increased the most, $21.16 \%$ and $89.87 \%$, respectively $(p<0.05)$, the TK content of stems inoculation with A13 increased the most by $37.75 \%$; The TN and TK content of leaves inoculation with A20 increased the most, $61.07 \%$ and $14.04 \%$, respectively $(p<0.05)$, and inoculation with A20 increased the TP content of leaves by up to $26.85 \%(p<0.05)$.

Inoculation with PGPR increased soil nutrient content (Fig. 3b). Inoculation with A13 increased the soil OM, TN and AN content the most, $39.58 \%, 49.18 \%$ and $47.62 \%$, respectively $(p<0.05)$; Inoculation with A20 increased soil TP content by $97.44 \%$; Inoculation with A36 increased the soil AP and TK content the most, $83.32 \%$ and $20.16 \%$, respectively $(p<0.05)$; Inoculation of three PGPRs increased the soil AK content significantly. Among them, the inoculation of A36 had the best effect, increasing by $98.10 \%(p<0.05)$.

\subsection{Effects of PGPR inoculation on seedlings and soil enzyme activities}

Inoculation with PGPR has a certain effect on plant enzyme activities (Fig. 4a, b, c, d, e, f). Inoculation with three PGPRs significantly increases POD and PSS by $23.15 \%-53.70 \%$ and $12.66 \%-27.29 \%$, respectively; Inoculation with A20 increased SOD activity by $16.76 \%(p<0.05)$; Inoculation with three PGPRs had a huge increase in CAT and PRO enzyme activities, the increase was $98.35 \%-128.69 \%$ and $283.67 \%-684.31 \%(p<0.05)$, among them, inoculation with A13 had the largest increase in CAT activity, while inoculation with A20 had the largest increase in PRO activity; inoculation with A36, A13 and A20 treatments reduced the MDA content by $36.22 \%, 56.12 \%$ and $13.78 \%$ respectively $(p<0.05)$.

Rhizosphere soil enzymes promote soil metabolism, change the form of soil nutrients, improve soil characteristics, and help increase the productivity of plant rhizosphere soil. PGPR inoculation had a certain effect on soil enzyme activity (Fig. 4g, h, i, j). Inoculation of A36, A13 and A20 significantly increased soil SA, increasing by $38.97 \%, 48.98 \%$ and $37.98 \%$ respectively $(p<0.05)$; At the same time, inoculation of the three PGPRs significantly increased soil APA by $22.48 \%, 38.95 \%$, and $21.48 \%(p<0.05)$; While only inoculation with A20 significantly increased CA and UA, by $10.45 \%$ and $23.00 \%$, respectively $(p<0.05)$.

\subsection{High-throughput sequencing analysis of microbial diversity in the rhizosphere soil}

\subsubsection{Effect of PGPR on the composition of the soil bacterial community}

Page 6/21 
The Venn diagram reflected the similarity and overlap of the samples. Following OTU clustering, separation, and elimination, a total of 3191 bacterial OTUs were obtained with a similarity of $97 \%$ (Fig. 5a). The unique bacterial OTUs of the A36, A13, A20, and CK groups were 174, 252, 192 , and 200, respectively. There were more unique bacteria in the soil treated with $A 13$, indicating that inoculation with $A 13$ provided a more favorable environment for microbial diversity. CK and A36 shared 1,550 OTUs, CK and A13 shared 1,731 OTUs, and CK and A20 shared 1,550 OTUs, indicating that the bacterial communities of the CK and A13 groups were more similar than those of the other groups.

Analysis of the sequencing results indicated that soil samples from the four treatment groups comprised a total of 27 phyla, 68 classes, 186 orders, 343 families, 641 genera, and 1310 species of soil bacteria. The bar chart shows the species composition of different groups at the phylum level (Fig. 5b). The most abundant bacterial phyla were Acidobacteria, Patescibacteria, Bacteroidetes, Chloroflexi, Gemmatimonadetes, Firmicutes, Actinobacteria, and Proteobacteria, which contributed almost $97.2 \%$ of the bacterial sequences. In the A36, A13, and A20 treatment groups, the relative abundance of Acidobacteria and Firmicutes was significantly lower than that of the CK group ( $p<0.01)$, while the opposite was observed regarding Patescibacteria $(p<0.01)$, the relative abundance of which after PGPR treatment was significantly higher than that of the CK group. There was no significant difference in the relative abundance of Proteobacteria. The relative abundance of Bacteroidetes in the A20 group was not significantly different from that of the CK group, but was significantly higher than that of the A36 and A13 groups ( $p<0.01$ ). The relative abundance of Gemmatimonadetes in the A36 group was not significantly different from that of the CK group, but it was significantly higher than that of A20 and A13 groups $(p<0.01)$. The relative abundance of Actinobacteria in the A36 and A20 groups was significantly higher than that of CK and A13 groups $(p<0.01)$.

The cluster analysis by heat map showed the community composition among the samples (Fig. $5 \mathrm{c}$ ). The sample clustering results showed that the A13 group and the CK group were relatively similar, and the A36 group and the A20 group were relatively similar. The A36, A20, and CK groups were clearly separated, indicating that the soil bacterial community changed after the addition of PGPR. The relative abundance of Arthrobacter in the CK group was 3.48\%, while the relative abundance of Arthrobacter was the highest in the A36 and A20 groups at $9.61 \%$ and $12.15 \%$, respectively, showing a significant increase $(p<0.05)$. In the A13 group, the relative abundance of Serratia was the highest at $5.42 \%$, while in the CK group, the relative abundance of Serratia was 0 , indicating that the relative abundance of Serratia increased significantly in the A13 group $(p<0.05)$.

\subsubsection{Relationship between soil bacterial community composition and soil environmental factors}

The relative abundance of bacterial dominant genera in 12 soil samples (Table 2), a total of 28 bacterial genera accounted for more than $1 \%$ of the total community of all treatment groups, and the bacterial dominant genera of each treatment group showed differences $(p<0.01)$. Compared with CK, inoculation with PGPR significantly reduced the relative abundance of Burkholderia-Caballeronia-Paraburkhol, Nocardioides and Blastococcus $(p<0.01)$, and significantly increased the relative abundance of Mesorhizobium, Tumebacillus, Conexibacter and Caenimonas $(p<0.01)$. There were also differences in the dominant genera of bacteria. 
Table 2

The community composition of soil bacteria after inoculation of different treatment groups

\begin{tabular}{|c|c|c|c|c|}
\hline Genera & A36 & A13 & A20 & CK \\
\hline Arthrobacter & $10.67 \pm 0.58 \mathrm{~B}$ & $5.03 \pm 0.73 \mathrm{C}$ & $14.00 \pm 1.00 \mathrm{~A}$ & $3.87 \pm 1.50 \mathrm{C}$ \\
\hline Ramlibacter & $6.47 \pm 0.49 \mathrm{~B}$ & $9.30 \pm 0.35 \mathrm{~A}$ & $6.20 \pm 0.20 \mathrm{~B}$ & $11.47 \pm 1.86 \mathrm{~A}$ \\
\hline Gemmatimonas & $9.53 \pm 0.57 \mathrm{AB}$ & $7.97 \pm 0.40 \mathrm{~B}$ & $5.87 \pm 0.21 \mathrm{C}$ & $9.93 \pm 1.10 \mathrm{~A}$ \\
\hline Bacillus & $6.63 \pm 0.31 \mathrm{~A}$ & $6.87 \pm 0.32 \mathrm{~A}$ & $9.70 \pm 2.25 \mathrm{~A}$ & $9.37 \pm 0.71 \mathrm{~A}$ \\
\hline Massilia & $8.10 \pm 0.40 \mathrm{~B}$ & $6.73 \pm 0.25 \mathrm{C}$ & $10.67 \pm 0.58 \mathrm{~A}$ & $7.97 \pm 0.23 \mathrm{~B}$ \\
\hline Burkholderia-Caballeronia-Paraburkhol & $12.30 \pm 2.56 \mathrm{~A}$ & $7.73 \pm 0.50 \mathrm{BC}$ & $8.87 \pm 1.47 \mathrm{AB}$ & $3.73 \pm 0.83 \mathrm{C}$ \\
\hline Sphingomonas & $11.67 \pm 1.15 \mathrm{~A}$ & $3.77 \pm 0.61 \mathrm{~B}$ & $12.00 \pm 0.00 \mathrm{~A}$ & $5.80 \pm 0.75 \mathrm{~B}$ \\
\hline Pseudolabrys & $7.27 \pm 0.06 \mathrm{~B}$ & $8.50 \pm 1.30 \mathrm{AB}$ & $9.87 \pm 0.23 \mathrm{~A}$ & $7.40 \pm 0.43 \mathrm{~B}$ \\
\hline Nocardioides & $12.33 \pm 1.53 \mathrm{~A}$ & $7.30 \pm 1.08 \mathrm{AB}$ & $4.37 \pm 0.67 \mathrm{~B}$ & $9.53 \pm 4.84 \mathrm{AB}$ \\
\hline Tumebacillus & $0.42 \pm 0.08 \mathrm{D}$ & $13.33 \pm 1.15 \mathrm{~B}$ & $3.17 \pm 0.23 \mathrm{C}$ & $16.33 \pm 0.58 \mathrm{~A}$ \\
\hline Serratia & $7.00 \pm 3.56 \mathrm{~B}$ & $22.33 \pm 0.58 \mathrm{~A}$ & $3.77 \pm 0.98 \mathrm{BC}$ & $0.01 \pm 0.00 \mathrm{C}$ \\
\hline Candidatus_Solibacter & $5.67 \pm 0.25 \mathrm{~B}$ & $9.43 \pm 1.43 \mathrm{~A}$ & $6.70 \pm 0.72 \mathrm{~B}$ & $11.67 \pm 0.58 \mathrm{~A}$ \\
\hline Flavisolibacter & $8.23 \pm 0.35 \mathrm{AB}$ & $7.20 \pm 0.66 \mathrm{~B}$ & $8.73 \pm 0.61 \mathrm{AB}$ & $9.70 \pm 1.15 \mathrm{~A}$ \\
\hline Streptomyces & $9.20 \pm 4.85 \mathrm{~A}$ & $11.00 \pm 1.00 \mathrm{~A}$ & $3.80 \pm 0.20 \mathrm{~A}$ & $6.83 \pm 0.25 \mathrm{~A}$ \\
\hline Mesorhizobium & $6.00 \pm 0.44 \mathrm{C}$ & $7.77 \pm 0.60 \mathrm{~B}$ & $8.07 \pm 0.31 \mathrm{~B}$ & $11.33 \pm 0.58 \mathrm{~A}$ \\
\hline Devosia & $8.37 \pm 0.06 \mathrm{AB}$ & $9.30 \pm 0.20 \mathrm{~A}$ & $7.87 \pm 0.29 \mathrm{~B}$ & $7.77 \pm 0.67 \mathrm{~B}$ \\
\hline Conexibacter & $4.43 \pm 0.72 \mathrm{C}$ & $10.63 \pm 0.63 \mathrm{~A}$ & $8.37 \pm 0.57 \mathrm{~B}$ & $10.00 \pm 0.91 \mathrm{AB}$ \\
\hline Terrabacter & $13.33 \pm 1.53 \mathrm{~A}$ & $2.63 \pm 0.31 \mathrm{~B}$ & $13.67 \pm 1.53 \mathrm{~A}$ & $3.87 \pm 1.07 \mathrm{~B}$ \\
\hline Bryobacter & $5.63 \pm 0.06 \mathrm{~A}$ & $33.27 \pm 43.08 \mathrm{~A}$ & $7.80 \pm 0.44 \mathrm{~A}$ & $8.63 \pm 0.40 \mathrm{~A}$ \\
\hline Lysobacter & $6.20 \pm 0.75 B$ & $9.87 \pm 1.20 \mathrm{~A}$ & $8.63 \pm 0.40 \mathrm{AB}$ & $8.50 \pm 1.21 \mathrm{AB}$ \\
\hline Rhodanobacter & $7.57 \pm 1.42 \mathrm{~B}$ & $10.93 \pm 1.10 \mathrm{~A}$ & $8.60 \pm 0.40 \mathrm{AB}$ & $6.40 \pm 1.06 \mathrm{~B}$ \\
\hline Micropepsis & $8.90 \pm 0.72 \mathrm{~A}$ & $9.40 \pm 0.56 \mathrm{~A}$ & $8.77 \pm 0.72 \mathrm{~A}$ & $6.10 \pm 0.62 \mathrm{~B}$ \\
\hline Caenimonas & $0.97 \pm 0.14 \mathrm{C}$ & $9.03 \pm 0.25 \mathrm{~B}$ & $9.23 \pm 0.35 \mathrm{~B}$ & $14.33 \pm 1.53 \mathrm{~A}$ \\
\hline Pullulanibacillus & $5.97 \pm 0.55 \mathrm{~B}$ & $2.77 \pm 0.25 \mathrm{~B}$ & $19.67 \pm 3.21 \mathrm{~A}$ & $4.60 \pm 0.56 \mathrm{~B}$ \\
\hline Pseudomonas & $9.87 \pm 7.22 \mathrm{~A}$ & $10.17 \pm 0.76 \mathrm{~A}$ & $11.83 \pm 2.84 \mathrm{~A}$ & $1.42 \pm 0.93 \mathrm{~A}$ \\
\hline Blastococcus & $13.67 \pm 3.05 \mathrm{~A}$ & $6.73 \pm 1.70 \mathrm{~B}$ & $4.70 \pm 1.24 \mathrm{~B}$ & $8.10 \pm 0.69 \mathrm{~B}$ \\
\hline Dyella & $3.50 \pm 0.90 \mathrm{~B}$ & $3.30 \pm 0.36 \mathrm{~B}$ & $25.00 \pm 1.73 \mathrm{~A}$ & $2.17 \pm 0.35 \mathrm{~B}$ \\
\hline Pantoea & $0.04 \pm 0.07 \mathrm{~A}$ & $32.93 \pm 42.50 \mathrm{~A}$ & $0.00 \pm 0.00 \mathrm{~A}$ & $0.40 \pm 0.43 \mathrm{~A}$ \\
\hline
\end{tabular}

The Pearson rank correlation was used to evaluate the relationship between the dominant bacterial genera and environmental factors (Table 3 ). There was a Pearson correlation between the dominant bacterial genera $(R A>1 \%)$ and environmental factors. The relative abundances of Arthrobacter, Bacillus, Massilia, Burkholderia-Caballeronia-Paraburkhol, Pseudolabrys, Streptomyces, Devosia, Bryobacter, Rhodanobacter, Micropepsis, Pseudomonas, Blastococcus and Pantoea were positively correlated with environmental factors, on the contrary, Ramlibacter, Sphingomonas, Nocardioides, Tumebacillus,Candidatus_Solibacter, Flavisolibacter, Mesorhizobium, Conexibacter and Caenimonas were significantly negatively correlated with environmental factors $(P<0.05)$, and other genera did not have any significant correlations (Table 2). The content of OM, TP, AP, TK and AK in soil may be the main driving force affecting the composition of bacterial communities. 
Table 3

Pearson correlation data between dominant bacterial genera and soil environmental factors after inoculation in different treatment groups

\begin{tabular}{|c|c|c|c|c|c|c|c|c|c|c|}
\hline Genera & OM & TN & AN & TP & AP & TK & AK & SA & CA & UA \\
\hline Arthrobacter & - & - & - & $0.951 *$ & - & - & - & - & - & - \\
\hline Ramlibacter & - & - & - & $-0.993^{\star *}$ & - & - & - & - & - & - \\
\hline Bacillus & - & - & - & - & - & - & - & - & 0.944 ** & - \\
\hline Massilia & - & $0.911^{\star}$ & - & - & - & - & - & - & - & - \\
\hline $\begin{array}{l}\text { Burkholderia- } \\
\text { Caballeronia- } \\
\text { Paraburkhol }\end{array}$ & - & - & - & - & $0.907 *$ & $0.903 *$ & - & - & - & - \\
\hline Sphingomonas & $-0.920^{\star}$ & - & - & - & - & - & - & - & - & - \\
\hline Pseudolabrys & - & - & - & - & - & - & - & - & - & $0.935^{\star}$ \\
\hline Nocardioides & - & - & - & - & - & - & - & - & - & $-0.949 * \star$ \\
\hline Tumebacillus & - & - & - & $-0.926^{\star}$ & - & - & - & - & - & - \\
\hline Candidatus_Solibacter & - & - & - & $-0.949 \star \star$ & - & - & - & - & - & - \\
\hline Flavisolibacter & - & - & - & - & - & - & - & $-0.906 *$ & - & $-0.988 * \star$ \\
\hline Streptomyces & - & $0.967 \star \star$ & - & - & - & - & - & - & - & - \\
\hline Mesorhizobium & - & - & - & - & - & - & $-0.950 \star \star$ & - & - & - \\
\hline Devosia & - & $0.913^{\star}$ & - & - & - & - & - & - & - & - \\
\hline Conexibacter & - & - & - & - & $-0.982^{\star \star}$ & $-0.945^{\star \star}$ & - & - & - & - \\
\hline Bryobacter & $0.982^{\star \star}$ & - & - & - & - & - & - & - & - & - \\
\hline Rhodanobacter & - & - & $0.972^{\star \star}$ & - & - & - & - & - & - & - \\
\hline Micropepsis & - & - & - & - & - & - & $0.972 * \star$ & $0.999 * *$ & - & - \\
\hline Caenimonas & - & - & - & - & $-0.950 * \star$ & 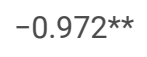 & - & - & - & - \\
\hline Pseudomonas & - & - & - & - & - & - & $0.965^{\star \star}$ & $0.937 *$ & - & - \\
\hline Blastococcus & - & - & - & - & - & - & - & - & - & 0.911 * \\
\hline Pantoea & $0.936 *$ & - & $0.916 *$ & - & - & - & - & - & - & - \\
\hline
\end{tabular}

\subsubsection{Diversity of bacterial community}

The coverage of the four soil libraries ranged from 0.987 to 0.990 (Fig. 6a), which was close to 1, indicating that the sequencing depth covered all the species of the soil sample, suggesting that the sequencing results represent the true situation of the soil bacteria. The order of the Chao index was A13 > CK > A20 > A36 (Fig. 6b), and except for the A13 group, there was no significant difference between the other groups. This indicated that the total number of soil bacterial species in the A13 group was higher than the other groups, and the community richness was also higher than the other groups. The Shannon index performance was: A13 >CK > A36 > A20 (Fig. $6 \mathrm{c}$ ). The larger the Shannon value, the higher the community diversity. The Simpson index was as follows: A20 > A36 > CK > A13 (Fig. 6d). The larger the Simpson value, the lower the community diversity. The A13 group index was significantly higher than other groups, indicating that the A13 group community diversity was higher.

Pearson analysis was used to analyze the correlation between the a diversity index and environmental factors (Fig. 6e). Chao and Shannon indexes were significantly positively correlated with TN and AN content $(p<0.05)$, and were extremely significantly positively correlated with OM content $(p<0.01)$. The Coverage index was significantly positively correlated with TP and AK content $(p<0.01)$. The Simpson index was positively correlated with TP content, but was negatively correlated with OM content. These findings indicate that PGPR has a crucial effect on soil nutrient cycling.

\subsubsection{Redundancy analysis of soil bacteria (RDA)}


The relationship between soil physicochemical properties and the relative abundances of dominant bacteria was assessed using RDA at the genus level (Fig. 7). The A20 sample was positively correlated with urease activity, but negatively correlated with TN and OM. A36 was positively correlated with TK and AP, and negatively correlated with AN, acid phosphatase activity, and CAT activity. A13 was positively correlated with OM and TN, and negatively correlated with TP. The RDA showed that the relative abundance of Arthrobacter, Sphingomonas was positively correlated with AP; the relative abundance of Arthrobacter and Sphingomonas was positively correlated with TP; the relative abundance of Arthrobacter was positively correlated with AK; and the relative abundance of Serratia and Tumebacillus was positively correlated with OM. The Monte-Carlo permutation test indicated that the AP, TP, AK, and OM indicators of the soil were significantly related to the bacterial community composition at the genus level (AP: pseudo-F =14.35, $p=0.004$; TP: pseudo-F = 7.96, $p=0.02$; AK: $p s e u d o-F=6.13, p=0.03$; OM: $p s e u d o-F=$ $10.30, p=0.009)$, indicating that soil AP, TP, AK, and OM were the main environmental factors influencing the composition of the microbial communities (at the genus level).

\section{Discussion}

Current agricultural production practices need to prioritize environmental sustainability, and thus the use of soil microorganisms has been suggested as a promising alternative to harmful pesticides and fertilizers, as well as for increasing crop yield (Wertz et al. 2007). PGPRs not only promote plant growth, control plant disease, and increase crop yield, but also exhibit strong rhizosphere colonization ability and specific microecological functions, and are thus potentially important biocontrol microorganisms (Schippers et al. 2003). Currently, the most representative PGPR genera that have been discovered are Pseudomonas, Enterobacter, Clostridium, Arthrobacter, Achromobacter, Micrococcus, Flavobacterium, Azospirillum, Azotobacter, and Bacillus (Cristiana et al. 2008; Swain and Ray, 2009). The three types of PGPR used in this study were P. mandelii, S. plymuthica, and P. koreensis. Pseudomonas rhizosphere bacteria have been extensively studied, though the two species used in the present experiment have been studied comparatively less. For instance, Ródenas et al. found that $P$. mandelii strain 29 could significantly increase mycorrhizal colonization (Navarro-Ródenas et al. 2016), while another study found that $P$. mandelii was associated with rice plant nitrogen fixation. Pseudomonas koreensis has not been reported on much, though P. koreensis Ps 9-14T has been isolated from the soil (Kwon et al. 2003), and another study discovered that $P$. koreensis could enhance the drought resistance of Helianthus annuus (Macleod et al. 2015). A P. koreensis JDM-2 strain was isolated from Eucommia ulmoides roots and exhibited ACC deaminase activity, and found that it had significant antibacterial effect on Bacillus subtilis (Gong 2011). Additionally, the genome sequence of $P$. koreensis CRS05-R5 strain has been analyzed (Lin et al. 2016). One study found that S. plymuthica BU09 had an obvious control effect on potato scab (Zhang and Liu, 2017), while another discovered that $S$. plymuthica A21-4 could improve cucumber quality and regulate the micro-ecological environment of cucumber rhizosphere soil (Ding et al. 2018). In this study, three treatment methods, including inoculation, compound inoculation, and non-inoculation, were used. The bacterial suspension was perforated and injected into the rhizosphere soil. The results showed that the seedling height, ground diameter, fresh weight, and dry weight of the $P$. sylvestris var. mongolica seedlings increased significantly after PGPR inoculation, which was consistent with previous results whereby the PGPRs Serratia proteamaculans 1-102 and Serratia liquefaciens 2-68 could promote the emergence and growth of corn (Pan et al. 1999). Thiery et al. found that the PGPR Bacillus amyloliquefaciens FZB 42 could promote cotton growth (Alavo et al. 2015), indicating that PGPRs can effectively promote plant growth.

In this study, the root length, surface area, number of root tips, and root volume of the seedlings increased significantly after PGPR inoculation, which may be related to the secretion of IAA by the three PGPR strains. A previous study showed that the effect of PGPR on root development is related to the secretion of IAA, as IAA can promote root growth and development, increase root surface area, and promote root metabolism (Glick 2014). Additionally, the inoculation of IAA-secreting PGPR on canola significantly increased the number of stems and branches (Asghar et al. 2004). The PGPR strains CA1001 and CA2004, which can secrete IAA, were inoculated into crops to enhance root and stem biomass (Chandra et al. 2018). Therefore, the increase in the root structure index of $P$. sylvestris var. mongolica seedlings may be mainly due to the IAA secreted by the PGPR. There is further evidence to illustrate this point; that is, among the three PGPR strains, A20 secreted the highest amount of IAA $(22.66 \mu \mathrm{g} / \mathrm{mL})$, and inoculation with strain A20 increased the root length, root surface area, root average diameter, and the number of root tips of the seedlings most significantly.

Plant enzymes such as SOD, POD, and CAT can effectively remove the reactive oxygen generated during metabolism, thus balancing the production and removal of active oxygen in the plant and preventing reactive oxygen from causing membrane peroxidation and other damage (Gururani and Upadhyaya, 2013; Habib et al. 2016). In this study, compared with CK, inoculation of A36, A20, and A13 significantly increased the POD, SOD, and CAT activity by $23.15-53.70 \%, 16.05-16.76 \%$, and $98.35-128.69 \%$, respectively $(p<0.05)$. This showed that the antioxidant enzyme activity of the $P$. sylvestris var. mongolica seedlings increased after inoculation with PGPR, and previous reports have suggested that SOD, POD, and CAT are important indicators of plant resistance induced by PGPR (Hahm et al. 2017; Uddin et al. 2015). This was confirmed by another study (Jha and Subramanian, 2013) that showed that after PGPR inoculation, the antioxidant enzyme activity of rice increased, thereby increasing plant resistance to salt stress.

PGPRs not only promote plant growth but also improve the rhizosphere environment of the plants. Soil enzyme activity can reflect the transformation ability of soil nutrients and the health of soil to a certain extent (Zhang et al. 2014). Inoculation with PGPRs was previously

Page $10 / 21$ 
found to significantly increase soil enzyme activity, total nitrogen, available nitrogen, and soil organic matter content (Ju et al. 2019). In this study, the same trend was observed. After inoculation with PGPR, the nutrient content of TP, AP, AK, and other nutrients in the rhizosphere soil of P. sylvestris var. mongolica increased significantly, and enzyme activities such as invertase and acid phosphatase were also increased. Some studies have demonstrated a close relationship between soil enzyme activity and soil available nutrients (Lian et al. 2011). For example, Li et al. found that soil CAT had a significant negative correlation with available phosphorus, and that urease had a significant positive correlation with available potassium (Ning et al. 2014). Another study found that soil CAT and phosphatase were significantly positively correlated with available nitrogen (Chen et al. 2014). In this study, after inoculation with the PGPRs, the enzyme activity of the rhizosphere soil increased, following which the content of available nutrients increased. The content of available nitrogen, phosphorus, and potassium increased the most, namely by $109.52 \%, 143.50 \%$, and $98.10 \%$, respectively. To some extent, the content of nitrogen, phosphorus, potassium, and other nutrients in the soil represents the potential fertility of the soil (Wang et al. 2015). Inoculation with PGPRs effectively improved the nutrient content of the rhizosphere soil of $P$. sylvestris var. mongolica and improved the ability of the plant to tolerate its external environment. In this study, it was found that after PGPR inoculation, in addition to the significant increase in biomass, the nutrient content and enzyme activity of the seedlings also increased. Many reports have suggested that PGPRs improve the biomass and the nutrient and enzyme activity of plants, thereby promoting plant growth and metabolism, such as in wheat and spinach (Akmak et al. 2007), pea (Akhtar 2014), and organically-grown raspberry (Orhan et al. 2006).

Inoculation with rhizosphere bacteria enriches some dominant bacterial groups, including some beneficial bacteria that participate in soil nutrient cycling, improves soil texture, and prevents and controls plant diseases. In this study, after inoculation with PGPR, some growthpromoting bacterial genera in the rhizosphere soil increased significantly, such as Arthrobacter, Ramlibacter, Gemmatimonas, Bacillus, and Serratia. Of these, Arthrobacter is a beneficial functional bacteria that can improve the IAA content and salt tolerance of plants (VelázquezBecerra et al. 2011). One of the important characteristics of the genus Bacillus is that it can produce spores with special resistance under adverse conditions, thus playing an active role in preventing plant diseases, improving plant resistance, and promoting plant growth (Oliveira et al. 2010; Probanza et al. 2002). Serratia is a beneficial bacteria that can tolerate heavy metals and repair plants, playing an important role in protecting the environment (76). Actinobacteria is a Gram-positive bacteria that can degrade cellulose and chitin as the main resource for soil nutrient supply. A recent study found that after applying nitrogen fertilizer, the abundance of Actinobacteria in the Gurbantünggüt Desert soil increased significantly (Huang et al. 2018), which is similar to the results of this study. After inoculation with PGPRs, the relative abundance of Actinobacteria in the rhizosphere soil was significantly positively correlated with soil AN content $(p<0.05)$. Chloroflexi is a Gram-negative bacteria that can potentially autotrophically metabolize through photosynthesis. This study found that after inoculation with PGPR, the relative abundance of Chloroflexi in the rhizosphere soil was significantly negatively correlated with soil AN content $(p<0.05)$, which is similar to the results of Ren et al. (Ren et al. 2020) who found that after inoculation with biochar + PGPR, the soil nitrogen content increased, while the abundance of Chloroflexi decreased. The cluster analysis results showed that the bacterial communities treated by strains A20 and A36 differed significantly from those treated with strains A13 and CK at the genus level, indicating that inoculation with strains A20 and A36 altered the soil bacterial community. The RDA results showed that soil AP, TP, AK, and OM indicators significantly affected the bacterial community composition, suggesting that there are the main environmental factors that contribute to the differences in microbial community composition.

\section{List Of Abbreviations}

AK, Available potassium; AN, Available nitrogen; AP, Available phosphorus; CAS, Chrome Azurol S; CAT, Catalase; IAA, Indole-3-acetic acid; MDA, Malondialdehyde; MSA, Mannitol Salt Agar; NB, Nutrient broth; OM,Organic matter; NBRIP, National Botanical Research Institute Phosphate; PCR, Polymerase Chain Reaction; PGPR, Plant Growth Promoting Rhizobacteria; POD, Peroxidase; PSS, Plant-soluble Sugar; PRO, Proline; PTP, Plant total protein ; SOD, Superoxide dismutase; TK, Total Potassium; TN, Total Nitrogen; TP, Total Phosphorus.

\section{Declarations}

\section{Ethics approval and consent to participate}

Not applicable

\section{Consent for publication}

Not applicable

\section{Availability of data and materials}

Not applicable

\section{Competing interests}


All the authors declare no conflict of interest.

\section{Funding}

This research was funded by the National Natural Science Foundation of China under grant numbers 31700564, 31670649, 31170597, and 31200484 and the National Key Research and Development Program under grant number 2017YFD0600101-6.

\section{Authors' contributions}

“Conceptualization, X-S. S. and X.D.; methodology, Q.S. and X.D.; software, Q.S., J-Y.L., J-K.W. and K.M.; resources, R-Q.S.; data curation, Q.S.; writing-original draft preparation, Q.S.; writing-review and editing, X.D. and X-S. S.; project administration, R-Q.S.; funding acquisition, R-Q.S. All authors have read and agreed to the published version of the manuscript.".

\section{Acknowledgements}

I hereby express my sincere thanks to my teacher Ruiqing Song for her help in the experiment and school life, and also appreciate the help given by Xun Deng and Xiaoshuang Song in the experiment.

\section{References}

1. Akhtar M S (2014) Effects of PGPR and antagonistic fungi on the growth, enzyme activity and Fusarium root-rot of pea. Arch Phytopathol Plant Prot 42: 138-148.

2. Akmak R, Erat M, Erdo An Ü, D Nmez M F (2007) The influence of plant growth-promoting rhizobacteria on growth and enzyme activities in wheat and spinach plants. J Plant Nutr Soil Sci 170: 288-295.

3. Alavo T B C, Boukari S, Fayalo D G, Bochow H (2015) Cotton fertilization using PGPR Bacillus amyloliquefaciens FZB42 and compost: Impact on insect density and cotton yield in North Benin, West Africa. Cogent Food Agric1.

4. Asghar H N, Zahir Z A, Arshad M (2004) Screening rhizobacteria for improving the growth, yield, and oil content of canola (Brassica napus L.). Aust J Agric Res 55: 187.

5. Barreto T R, Silva A C M D, Soares A C F, Souza J T D (2008) Population densities and genetic diversity of actinomycetes associated to the rhizosphere of Theobroma cacao. Braz J Microbiol 39: 464-470.

6. Bric J M, Bostock R M, Silverstone S E (1991) Rapid in situ assay for indoleacetic acid production by bacteria immobilized on a nitrocellulose membrane. Appl Environ Microb 57: 535-538.

7. Caporaso J G, Kuczynski J, Stombaugh J, Bittinger K, Bushman F D, Costello E K, Fierer N, Pe A A G, Goodrich J K, Gordon J I (2010) QIIME allows analysis of high-throughput community sequencing data 7: 335-336.

8. Cappuccino J (2010) Microbiology:A Laboratory Manual: International Edition. Addison-Wesley.

9. Chandra S, Askari K, Kumari M (2018) Optimization of indole acetic acid production by isolated bacteria from Stevia rebaudiana rhizosphere and its effects on plant growth. J. Genet. Eng. Biotechnol 16: 581-586.

10. Chen S X (2006) High-sensitive Detection Method for Siderophores from Pseudomonas. Microbiol 33: $122-127$.

11. Chen ZF, Liu JF, Wu ZY (2014) Soil Physicochemical Properties and Enzyme Activities at Different Elevation Gradients. Journal of Hebei North University (Natural Science Edition) 1: 38-42.

12. Cocking E C (2003) Endophytic colonization of plant roots by nitrogen-fixing bacteria. Plant \& Soil 252: $169-175$.

13. Compant S, Clément C, Sessitsch A (2010) Plant growth-promoting bacteria in the rhizo- and endosphere of plants: Their role, colonization, mechanisms involved and prospects for utilization. Soil Biol Biochem 42: 669-678.

14. Cristiana, Felici, And, Lorenzo, Vettori, And, Enrico, Giraldi, And, Laura (2008) Single and co-inoculation of Bacillus subtilis and Azospirillum brasilense on Lycopersicon esculentum: Effects on plant growth and rhizosphere microbial community. Appl Soil Ecol 40: $260-270$.

15. Determination of organic matter in forest soil and calculation carbon-nitrogen ratio. People's Republic of China Forestry Industry Standard. LY/T 1237-1999.

16. Ding FL, Li M, Liu DP, Zhang ZL, Sun YQ, Piao FZ, Shen SS (2018) Effects of plant rhizosphere growth-promoting bacterium Serratia pichii A21-4 on cucumber growth and soil microecology. China Veg 5: 36-41.

17. Edgar R C (2013) UPARSE: highly accurate OTU sequences from microbial amplicon reads. Nat Methods 10: 996.

18. Feng-Min L I (2002) The roles of root exudation on rhizosphere nutrient and rhizosphere microorganisms. Acta Bot Boreali-Occident Sin 22(3): 693-702.

19. Fiorentino N, Ventorino V, Woo S L, Pepe O, De Rosa A, Gioia L, Romano I, Lombardi N, Napolitano M, Colla G, Rouphael Y (2018) Trichoderma-Based Biostimulants Modulate Rhizosphere Microbial Populations and Improve N Uptake Efficiency, Yield, and Nutritional 
Quality of Leafy Vegetables. Front Plant Sci 9: 743.

20. Galkiewicz J P, Kellogg C A (2008) Cross-kingdom amplification using bacteria-specific primers: complications for studies of coral microbial ecology. Appl Environ Microbiol 74: 7828-7831.

21. Glick B R (2014) Bacteria with ACC deaminase can promote plant growth and help to feed the world. Microbiol Res 169: $30-39$.

22. Gong F J (2011) Isolation and antibacterial activity of ACC deaminase-containing endophytic bacteria from Eucommia ulmoides Oliver. Microbiol China 38: 1526-1532.

23. Gururani M A, Upadhyaya C P (2013) Plant Growth-Promoting Rhizobacteria Enhance Abiotic Stress Tolerance inSolanum tuberosumThrough Inducing Changes in the Expression of ROS-Scavenging Enzymes and Improved Photosynthetic Performance. J Plant Growth Regul 32: 245-258.

24. Habib S H, Kausar H, Saud H M (2016) Plant Growth-Promoting Rhizobacteria Enhance Salinity Stress Tolerance in Okra through ROSScavenging Enzymes. Biomed Res Int 2016: 6284510-6284547.

25. Hahm M, Son J, Hwang Y, Kwon D, Ghim S (2017) Alleviation of Salt Stress in Pepper (Capsicum annum L.) Plants by Plant GrowthPromoting Rhizobacteria. J Microbiol Biotechn 27: 1790-1797.

26. Huang KC, Shen YY, Xu GP, Huang YQ, Zhang DN, Sun YJ, Li YQ, He W, Zhou LW (2018) Effects of Reclamation on Soil Nutrients and Microbial Activities in the Huixian Karst Wetland in Guilin. Environ Sci 39: 1813-1823.

27. G S, G. M Z, D. G Z (2002) Actinomycetes in the Rhizosphere of Barley Grown on Acid Soddy Podzolic Soil. Microbiol 71: $455-459$.

28. Jha Y, Subramanian R B (2013) Paddy plants inoculated with PGPR show better growth physiology and nutrient content under saline conditions. Chil J Agr Res 73: 213-219.

29. Ju W, Liu L, Fang L, Cui Y, Duan C, Wu H (2019) Impact of co-inoculation with plant-growth-promoting rhizobacteria and rhizobium on the biochemical responses of alfalfa-soil system in copper contaminated soil. Ecotoxicol Environ Saf 167: 218-226.

30. Kloepper J W, Leong J, Teintze M, Schroth M N (1980) Enhanced plant growth by siderophores produced by plant growth-promoting rhizobacteria. Nature 286: 885-886.

31. Kloepper J W, Rodríguez-Kábana R, Mcinroy J A, Collins D J (1991) Analysis of populations and physiological characterization of microorganisms in rhizospheres of plants with antagonistic properties to phytopathogenic nematodes. Plant \& Soil 136: 95-102.

32. Koo S, Cho K (2009) Isolation and characterization of a plant growth-promoting rhizobacterium, Serratia sp. SY5. J Microbiol Biotechn 19: 1431.

33. Kwon S W, Kim J S, Park I C, Yoon S H, Park D H, Lim C K, Go S J (2003) Pseudomonas koreensis sp. nov., Pseudomonas umsongensis sp. nov. and Pseudomonas jinjuensis sp. nov., novel species from farm soils in Korea. Int J Syst Evol Micr 53: 21-27.

34. Lian L, Xie L, Chen J, Zheng L, Lin (2011) Colonization of biocontrol strain EN5 and its effects on rhizosphere soil microbial communities. Plant Prot 37: 31-35.

35. Lin H, Hu S, Liu R, Chen P, Ge C, Zhu B, Guo L (2016) Genome Sequence of Pseudomonas koreensis CRS05-R5, an Antagonistic Bacterium Isolated from Rice Paddy Field. Front Microbiol 7.

36. Liu Z (2005) Research Advances in Root Exudates and Rhizosphere Microorganisms of Forest Trees. World Forestry Research 18: 25-31.

37. Macleod K, Rumbold K, Padayachee K (2015) A Systems Approach to Uncover the Effects of the PGPR Pseudomonas koreensis on the Level of Drought Stress Tolerance in Helianthus Annuus. Procedia Environ Sci 29: 262-263.

38. Magoc T, Salzberg S L (2011) FLASH: fast length adjustment of short reads to improve genome assemblies. Bioinf 27: $2957-2963$.

39. Myresiotis C K, Vryzas Z, Papadopoulou-Mourkidou E (2012) Biodegradation of soil-applied pesticides by selected strains of plant growthpromoting rhizobacteria (PGPR) and their effects on bacterial growth. Biodegrad 23: 297-310.

40. Navarro-Ródenas A, Berná L M, Lozano-Carrillo C, Andrino A, Morte A (2016) Beneficial native bacteria improve survival and mycorrhization of desert truffle mycorrhizal plants in nursery conditions. Mycorrhiza 26: 769-779.

41. Ning L I, Zhang J Y, Zhi-Guo S I (2014) Correlation Analysis of Enzyme Activity and Physi-chemical Characteristics of Soil in City Parks of Zhengzhou. J. West China For Sci 43.

42. Nitrogen determination methods of forest soils. People's Republic of China Forestry Industry Standard. LY/T 1228-2015.

43. Oliveira J R G D, Moraes T A D L, Melo N F D, Yano-Melo A M (2010) Fungos micorrízicos arbusculares e rizobactérias promotoras de crescimento na aclimatização de zingiber spectabile. Bragantia 69: 687-694.

44. Orhan E, Esitken A, Ercisli S, Turan M, Sahin F (2006) Effects of plant growth promoting rhizobacteria (PGPR) on yield, growth and nutrient contents in organically growing raspberry. Sci Hortic-Amsterdam 111: 38-43.

45. Pan B, Bai Y M, Leibovitch S, Smith D L (1999) Plant-growth-promoting rhizobacteria and kinetin as ways to promote corn growth and yield in a short-growing-season area. Eur J Agron 11: 179-186. 
46. Patterson B D, Macrae E A, Ferguson I B (1984) Estimation of hydrogen peroxide in plant extracts using titanium(IV). Anal Biochem 139: 487-492.

47. Pérez-Miranda S, Cabirol N, George-Téllez R, Zamudio-Rivera L S, Fernández F J (2007) O-CAS, a fast and universal method for siderophore detection. J Microbiol Meth 70: 127-131.

48. Phosphorus determination methods of forest soils. People's Republic of China Forestry Industry Standard. LY/T $1232-2015$.

49. Potassium determination methods of forest soils. People's Republic of China Forestry Industry Standard. LY/T 1234-2015.

50. Probanza A, Lucas García J A, Ruiz Palomino M, Ramos B, Gutiérrez Mañero F J (2002) Pinus pinea L. seedling growth and bacterial rhizosphere structure after inoculation with PGPR Bacillus (B. licheniformis CECT 5106 and B. pumilus CECT 5105). Appl Soil Ecol 20: 7584.

51. Qi JU, Yin DC, Song RQ (2019) Effects of Suillus luteus on Salt Resistance of Pinus sylvestris var. mongolica Seedlings. J. Shenyang Agric Univ 50: 108-113.

52. Ren H, Huang B, Fernández-García V, Miesel J, Yan L, Lv C (2020) Biochar and Rhizobacteria Amendments Improve Several Soil Properties and Bacterial Diversity. Microorganisms 8: 502.

53. R.Rai (1991) Strain-specific salt tolerence and chemotaxis of Azospirillum brasilense and their associative $\mathrm{N}$-fixation with finger millet in saline calcareous soil Plant \& Soil. 137: 55-59.

54. Schippers B A, Bakker A W, Bakker P A H M (2003) Interactions of Deleterious and Beneficial Rhizosphere Microorganisms and the Effect of Cropping Practices. Annu Rev Phttopathol 25: 339-358.

55. Shekhar N C (1999) An efficient microbiological growth medium for screening phosphate solubilizing microorganisms. Fems Microbiol Lett 170: 270 .

56. Shin S H, Yong L, Lee S E, Yang N W, Rhee J H (2001) CAS agar diffusion assay for the measurement of siderophores in biological fluids. J Microbiol Meth 44: 95.

57. Singh R P, Jha P N (2016) The Multifarious PGPR Serratia marcescens CDP-13 Augments Induced Systemic Resistance and Enhanced Salinity Tolerance of Wheat (Triticum aestivum L.). Plos One 11:e155026.

58. Stefan Kurze, Hubert Bahl (2001) Biological Control of Fungal Strawberry Diseases by Serratia plymuthica HRO-C48. Plant Dis 85.

59. Sreevidya M, Gopalakrishnan S, Kudapa H and Varshney R K (2016) Exploring plant growth-promotion actinomycetes from vermicompost and rhizosphere soil for yield enhancement in chickpea. Braz J Microbiol 47: 85-95.

60. Swain M R, Ray R C (2009) Biocontrol and other beneficial activities of Bacillus subtilis isolated from cowdung microflora. Microbiol Res 164: 121-130.

61. Uddin I, Bano A, Masood S (2015) Chromium toxicity tolerance of Solarium nigrum L. and Parthenium hysterophorus L. plants with reference to ion pattern, antioxidation activity and root exudation. Ecotoxicol Environ Saf 113: 271-278.

62. Uroz S, Oger P, Tisserand E, Cébron A, Turpault M P, Buée M, De Boer W, Leveau J H J and Frey-Klett P (2016) Specific impacts of beech and Norway spruce on the structure and diversity of the rhizosphere and soil microbial communities. Sci Rep-UK 6.

63. Velázquez-Becerra C, Macías-Rodríguez L I, López-Bucio J, Altamirano-Hernández J, Flores-Cortez I, Valencia-Cantero E (2011) A volatile organic compound analysis from Arthrobacter agilis identifies dimethylhexadecylamine, an amino-containing lipid modulating bacterial growth and Medicago sativa morphogenesis in vitro. Plant \& Soil 339: 329-340.

64. Vessey J K (2003) Plant growth promoting rhizobacteria as biofertilizers. Plant \& Soil 255: 571-586.

65. Wang SQ, Wei SJ, Yang TT, Li QM, Tu GQ, Ni GR, Pan XH (2015) Effects of Streptomyces jd211 on the growth promotion of rice seedlings and the diversity of soil bacteria. Acta Pedologica Sinica 52: 673-681.

66. Wertz S, Degrange V, Prosser J I, Poly F, Commeaux C, Guillaumaud N, Le Roux X (2007) Decline of soil microbial diversity does not influence the resistance and resilience of key soil microbial functional groups following a model disturbance. Environ Microbiol 9: 22112219.

67. Wu Z Y, Lin W X, Chen Z F, Fang C X, Shen L H (2013) Characteristics of soil microbial community under different vegetation types in Wuyishan National Nature Reserve, East China. Chin. J. Appl. Ecol 24: 2301-2309.

68. Xiang Z (2006) Improvement and Analysis in Over- siderophores Production Bacteria Filtrating and Detecting. Microbiol 33: 95-98.

69. Yang C, Bai L, Liang RT, Jie WG, Sun HB, Cai BY (2019) Effect of Funneliformis mosseae on the Continuous Cropping Soybean Root and Soil Rhizosphere Enzymes Activities. Chinese Agricultural Science Bulletin 35: 91-98.

70. Yayou L, Di Y U, Ding F, Fengzhi P, Shen S (2016) Effects of PGPR Strain A21-4 on Growth and Rhizosphere Soil Charactors of Pepper in Field. Chinese Journal of Biological Control 32: 86-92.

71. Yue D, Zhang Y (2009) The Biocontrol Effects of Pseudomonas in the Rhizosphere of Tomatoes. Acta Agriculturae Boreali-Sinica 24: 210213. 
72. Zhang F, Li L (2003) Using competitive and facilitative interactions in intercropping systems enhances crop productivity and nutrient-use efficiency. Plant \& Soil 248: 305-312.

73. Zhang F, Huo Y, Cobb A B, Luo G, Zhou J, Yang G, Wilson G W T, Zhang Y (2018) Trichoderma Biofertilizer Links to Altered Soil Chemistry, Altered Microbial Communities, and Improved Grassland Biomass. Front Microbiol 9: 848.

74. Zhang Q S, Yu X T (1990) Study on the Seasonal Dynamics of Soil Microbes in Mixed Forest Plantation of Cunninghamia lanceolata. Acta Ecologica Sinica 2: 121-126.

75. Zhang Q Y, Gang L I, Yang Z G, Wang L J, Wang H, Chang H, Yang D L, Zhao J N (2014) Effects of transgenic soybean on enzyme activities and nutrients in rhizosphere soil. Chin J Oil Crop Sci 36: 409-413.

76. Zhang Y, Zhu Y, Yao T, Qi J, Rong LY (2013) Study on the interaction effect of four growth-promoting strains isolated from forage rhizosphere (PGPR). Acta Prataculturae Sinica 22: 29-37.

77. Zhang ZJ, Liu Q (2017) Screen and Identification of a Serratia plymuthica BU09 and Its Inhibition Effect against S.scabies. Journal of Anhui Agri 45: 123-124.

78. Zhu Z, Bingqi W U, Ding Y, Chen X, Zhang C, Binghai D U, Wang Y (2012) The Growth-promoting Effect and System Resistance Induction of Brevibacillus brevis DZQ3 to Tobacco. Chinese Tobacco Science 33: 92-106.

\section{Figures}

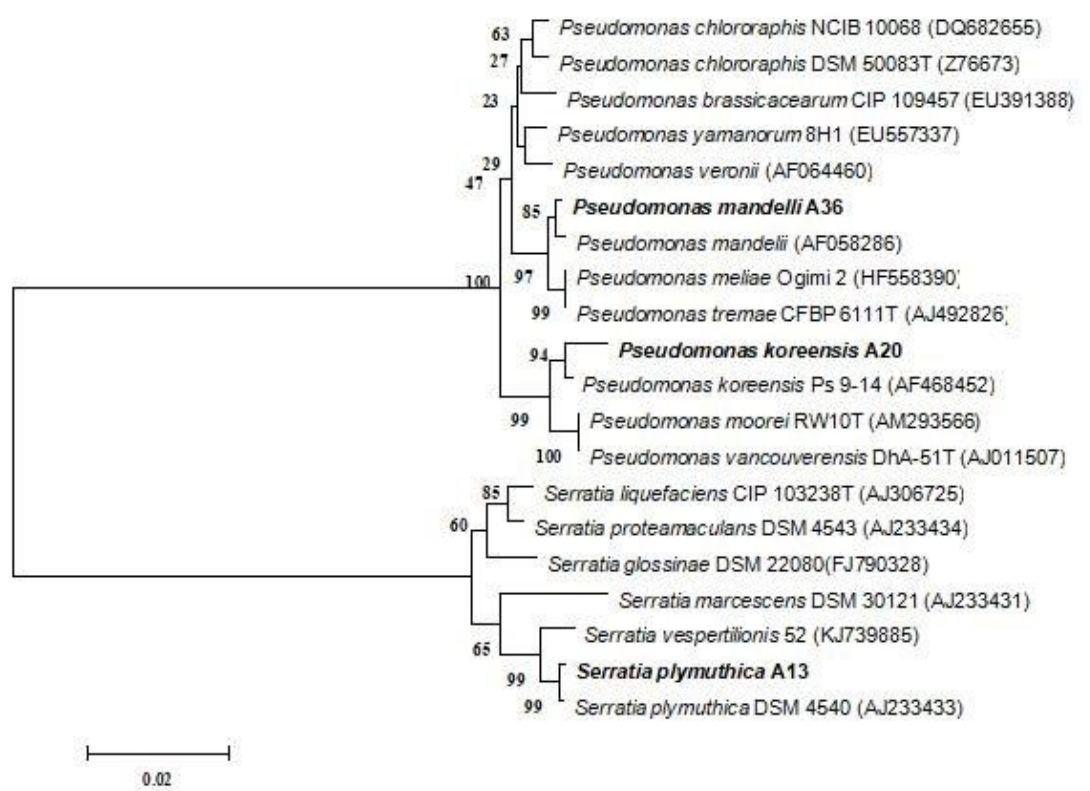

\section{Figure 1}

Phylogenetic tree of bacterial 16S rRNA gene sequences. Bootstrap values are indicated at tree branching points. 

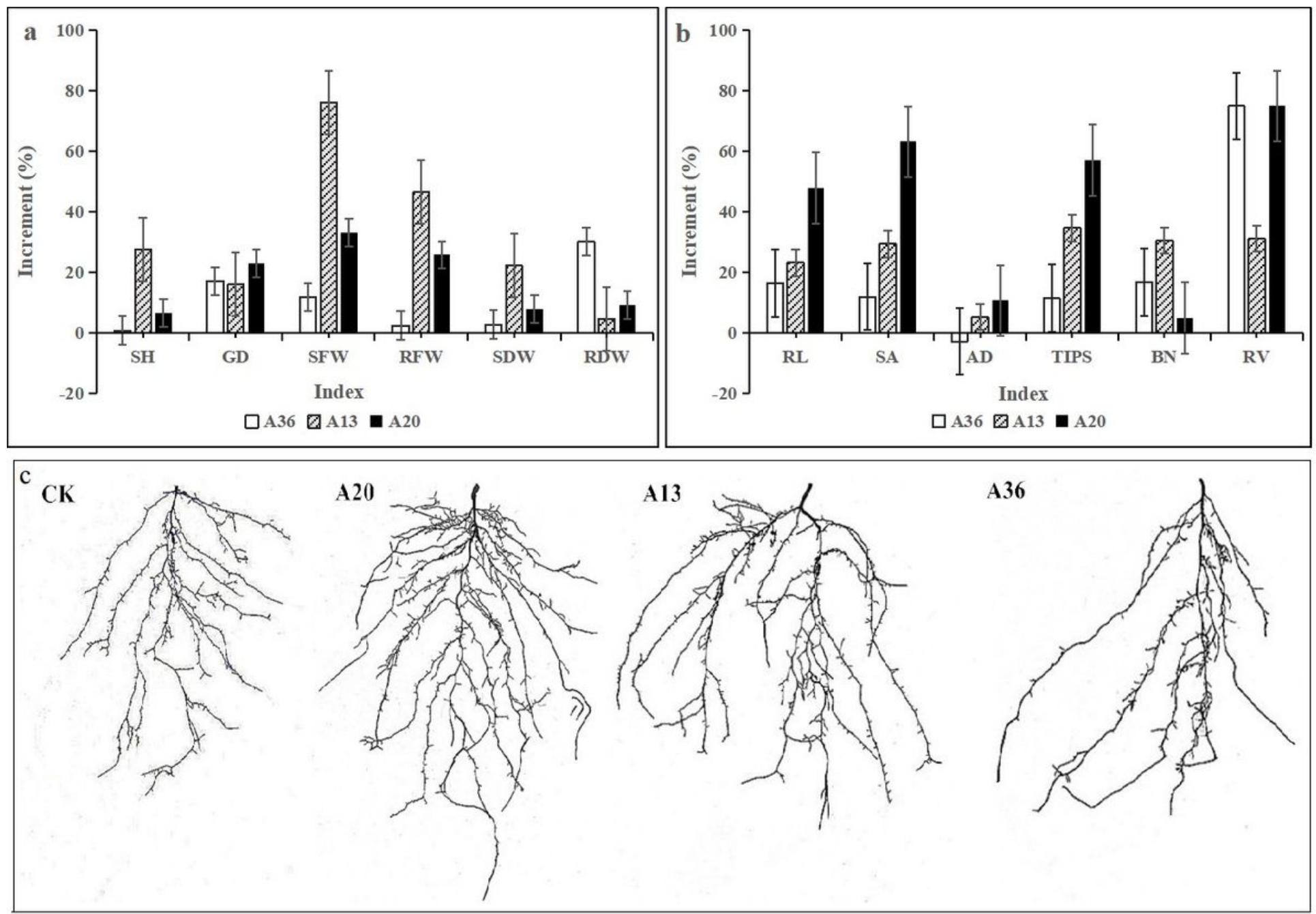

Figure 2

Biomass and root system indexes after inoculation in different treatment groups (a), biomass index (b), root system index (c), root system. 

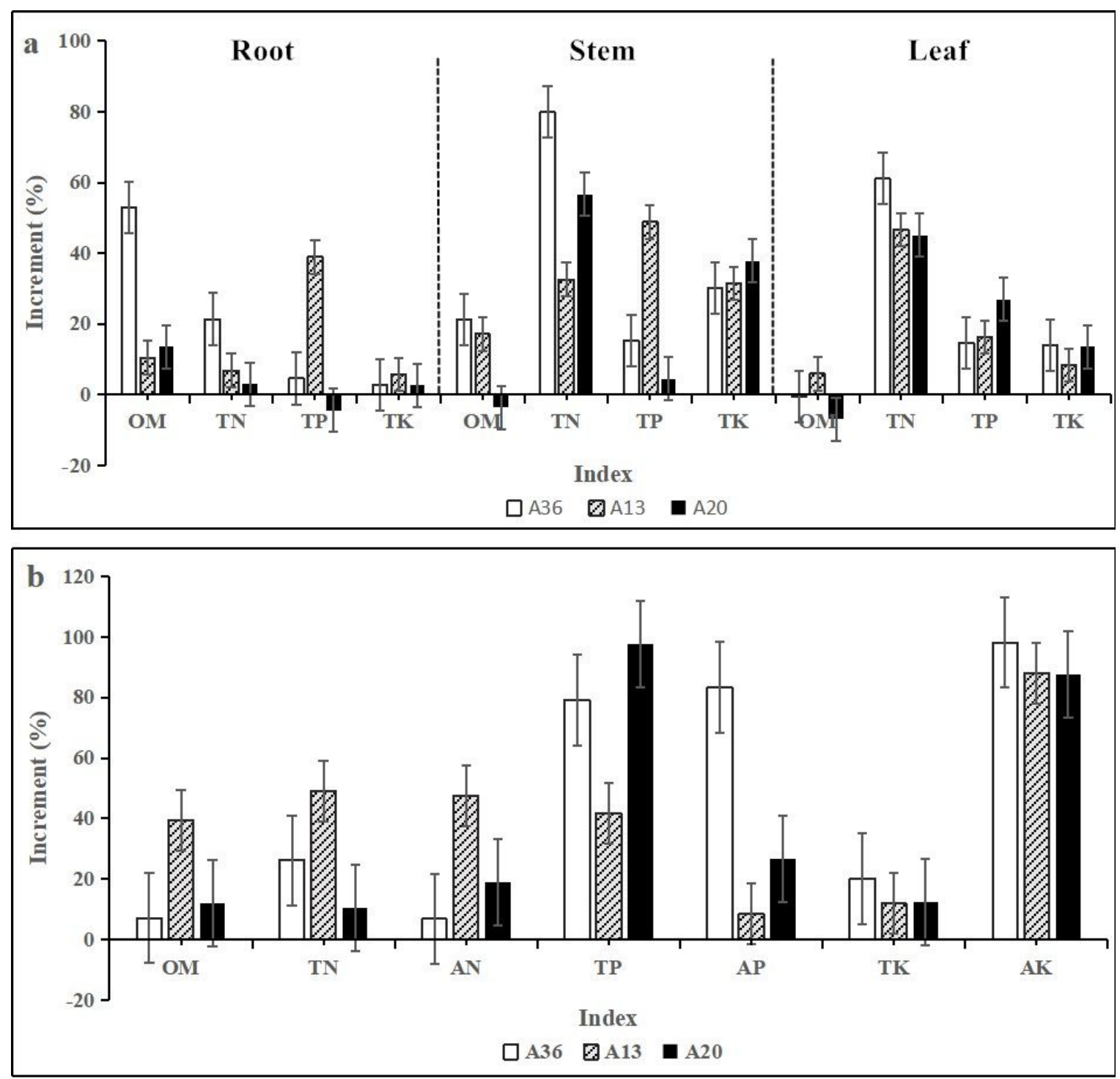

Figure 3

Nutrient index of seedlings and soil after inoculation in different treatment groups. (a), The nutrient index of seedling (b), the nutrient index of soil. 

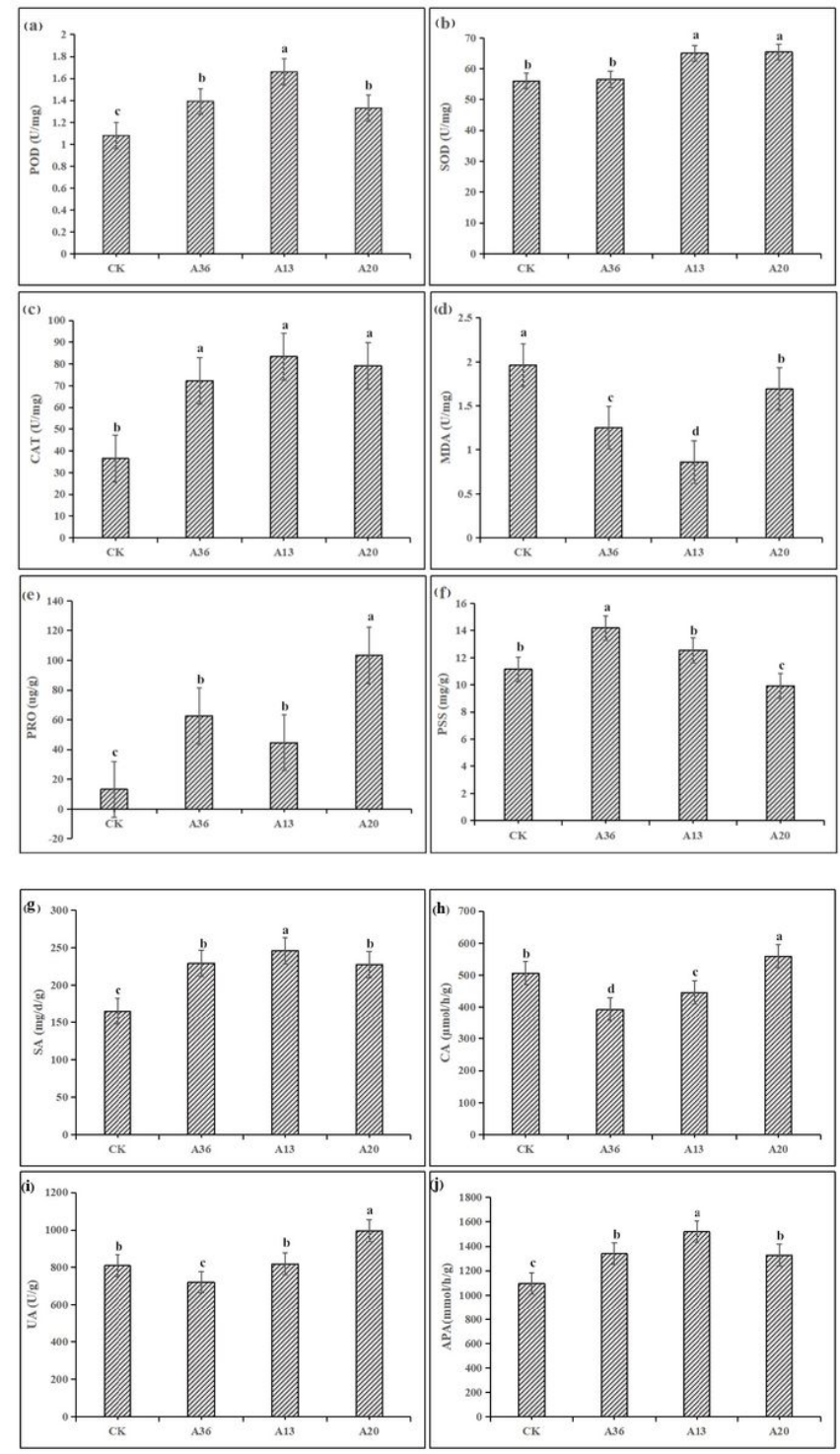

\section{Figure 4}

The enzyme activity indexes of seedlings and soil after inoculation in different treatment groups. (a) POD index of seedling, (b) SOD nutrient index of seedling, (c) CAT index of seedling, (d) MDA index of seedling, (e) PRO index of seedling, (f) PSS index of seedling, (g) SA index of soil, (h) CA index of soil, (i) UA index of soil, (j) APA index of soil. 
A20

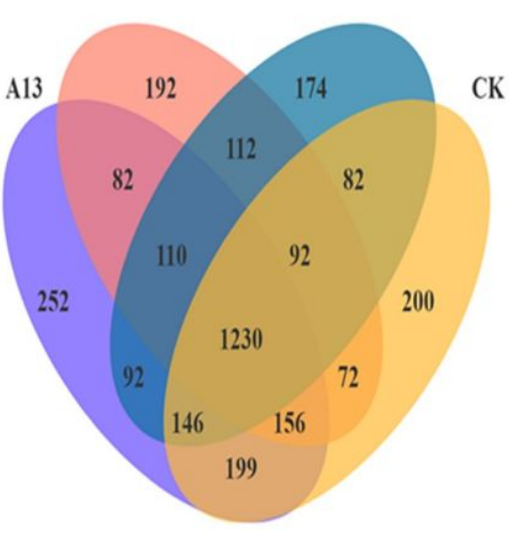

b

Community barplot analysis

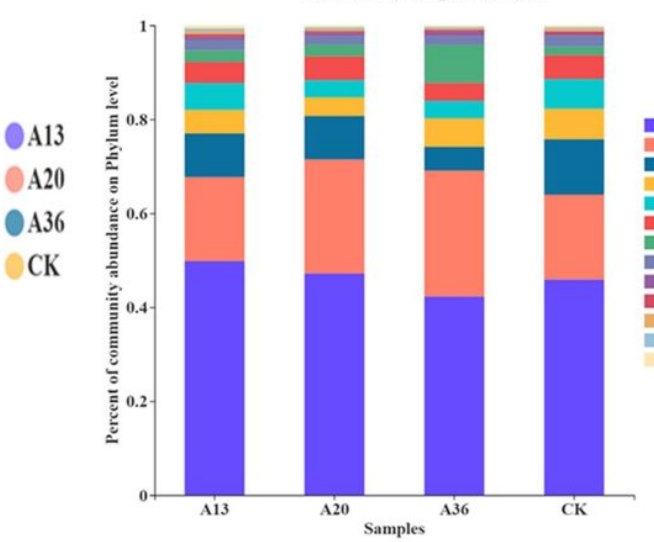

- Proteobacteria

Actinobacteria

Gemmatimonade

Geridobacteria

Bacteroidetes

Patescibacteria

Chloroflexi

Cyanobacteria

Verrucomicrobia

Armatimonadete

Planctomycetes

c
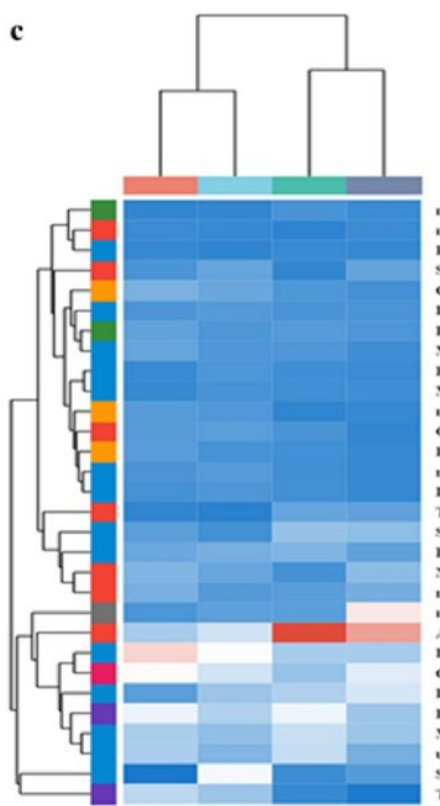

norank_fCChitimophagaceae

Bradyrbirobium

Stroptomyces
Candidatus_Solbacte

Devoria

Favibelibacter

Mesorbizobium
Rhodanobacter

Rhodanobacter

Silcropepsi:

norank_ron

Conexibacter

norank \& Nicropepaceae

Lyobacter

Terrabacter

Sphingomonas

Pseudelabrys

Nocardioides

nomak of

Arthrobacter

Rambibacter

Gerkmantonas

Burkholdenta

Massitia

unclassine

$\begin{array}{llll}C K & A 13 & A 20 & A 36\end{array}$

Sumebacillus

EK

표 A13

슬 20

수으

Bacteroidetes

Actinobacteria

Proteobacteria

Acidobacteria

Patescibacteria

-Gemmatimonadetes

- Firmicutes

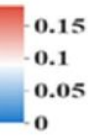

\section{Figure 5}

The species composition of soil after inoculation in different treatment groups (a) Species venn diagram, (b) Community Bar diagram (c) Community Heatmap diagram, the species cluster relationship is shown on the left tree. The sample cluster relationship is displayed on the top tree. 

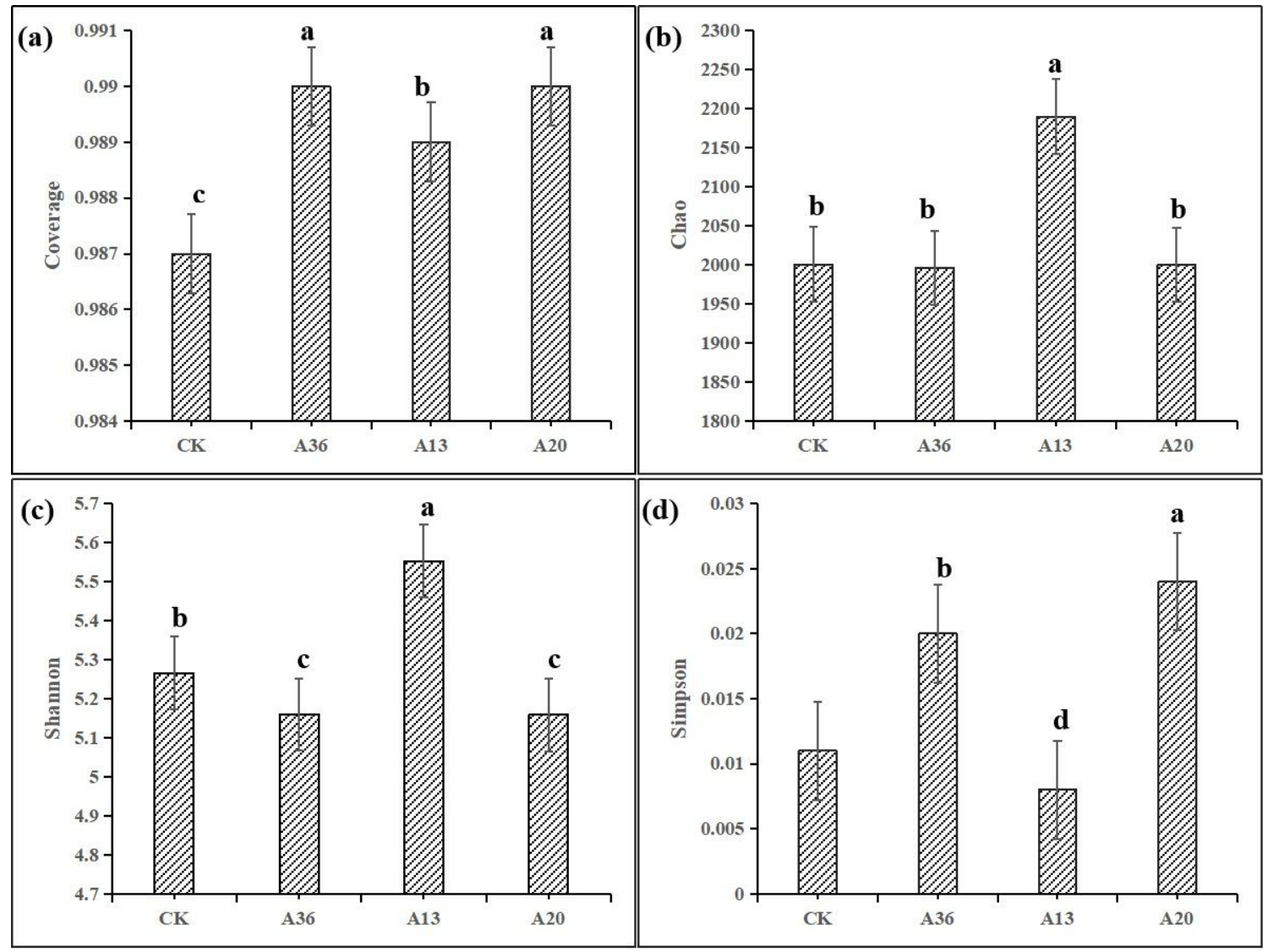

(e)

\begin{tabular}{ccccc}
\hline Index & Coverage & Chao & Simpson & Shannon \\
\hline OM & & $0.937^{* *}$ & $0.873^{*}$ & $0.993^{* *}$ \\
TN & - & $0.855^{*}$ & - & $0.817^{*}$ \\
AN & - & $0.922^{*}$ & - & $0.828^{*}$ \\
TP & $0.961^{* *}$ & - & $0.818^{*}$ & - \\
AK & $0.955^{* *}$ & - & - & - \\
\hline
\end{tabular}

\section{Figure 6}

The relationship between soil bacterial alpha diversity index and environmental factors after inoculation of different treatment groups. (A) Coverage index, (b) Chao index, (c) Simpson index, (d) Shannon index, (e) a diversity index and its Pearson correlation with environmental factors, * Significantly correlated at the 0.05 level, ** Significantly correlated at the 0.01 level. 


\section{RDA on Genus level}

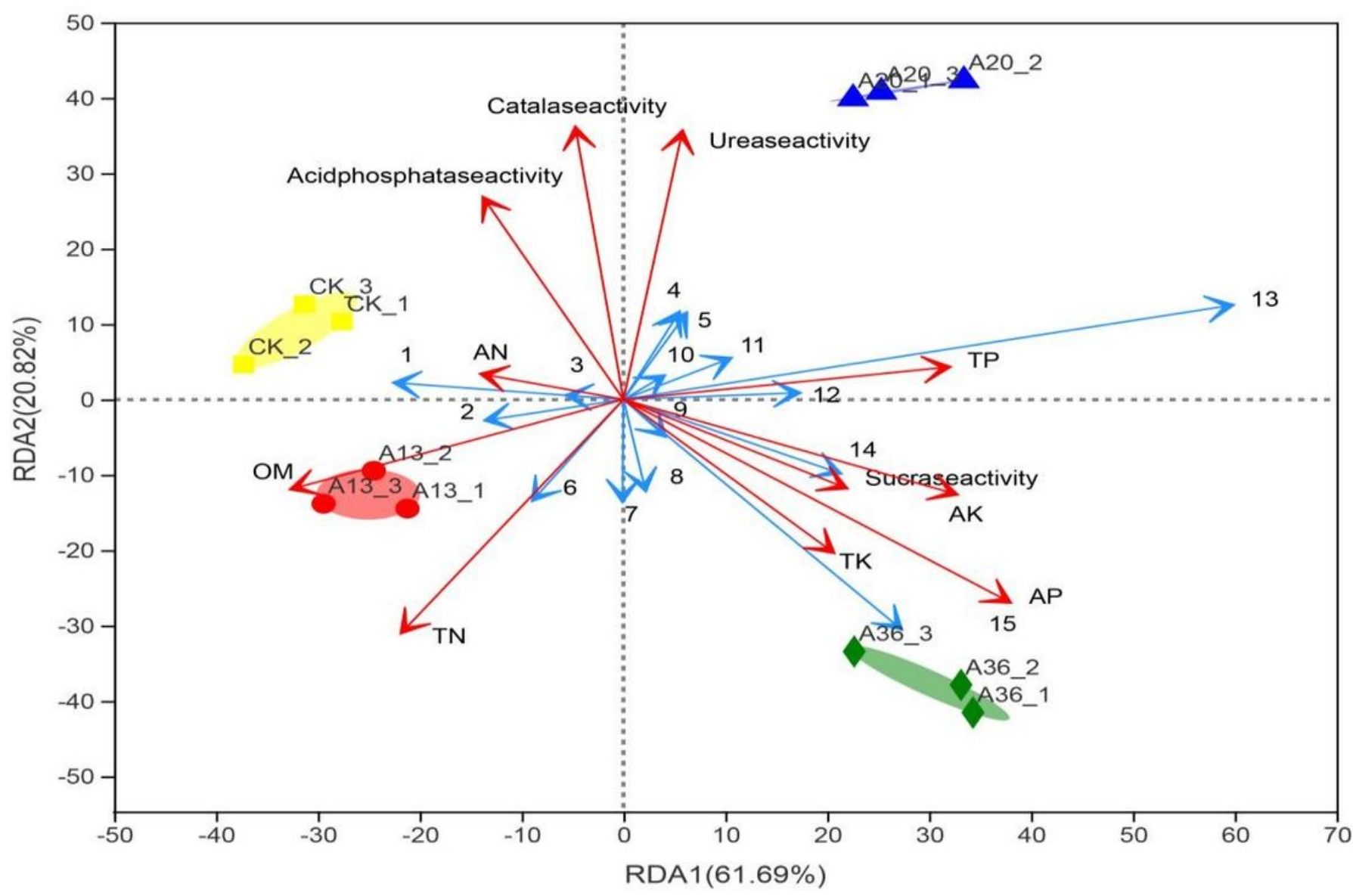

\section{Figure 7}

Redundancy analysis of the composition of the soil bacterial community and soil physiochemical properties at the genus level. Bacterial genera are represented by blue lines, and soil physiochemical properties (environmental factors) are represented by red lines ( 1 : Tumebacillus, 2:

Ramlibacter, 3: Candidatus, 4: Brevundimonas, 5: Bacillus, 6: Serratia, 7: Gemmatimonas, 8: Nocardioides, 9: norank_f_67-14, 10: Pseudolabrys, 11: Massilia, 12: Sphingomonas, 13: Arthrobacter, 14: Paraburkholderia, and 15:Saccharimonadales). 